\title{
Quantitative Precipitation Estimation with Operational Polarimetric Radar Measurements in Southern China: A Differential Phase-Based Variational Approach
}

\author{
HaO Huang, ${ }^{\text {a,b,c }}$ Kun ZhaO, ${ }^{\text {a,b }}$ GuIfu Zhang, ${ }^{\text {a,c }}$ QInG Lin, ${ }^{\text {d,a }}$ LONG Wen, ${ }^{a}$ GANG Chen, ${ }^{\text {a }}$ \\ ZHengwei YANG, ${ }^{\mathrm{a}}$ MingJun WANG, ${ }^{\mathrm{a}}$ AND DONGMING Hu ${ }^{\mathrm{e}}$ \\ ${ }^{a}$ Key Laboratory for Mesoscale Severe Weather, Ministry of Education, and School of Atmospheric Sciences, Nanjing University, Nanjing, China \\ ${ }^{\mathrm{b}}$ State Key Laboratory of Severe Weather, and Joint Center for Atmospheric Radar Research of China Meteorological \\ Administration-Nanjing University, Chinese Academy of Meteorological Sciences, Beijing, China \\ c School of Meteorology, and Advanced Radar Research Center, University of Oklahoma, Norman, Oklahoma, \\ ${ }^{\mathrm{d}}$ Guangdong Meteorological Observatory, Guangzhou, Guangdong, China \\ ${ }^{\mathrm{e}}$ Guangzhou Meteorological Observatory, Guangzhou, China
}

(Manuscript received 23 August 2017, in final form 8 March 2018)

\begin{abstract}
Quantitative precipitation estimation (QPE) with polarimetric radar measurements suffers from different sources of uncertainty. The variational approach appears to be a promising way to optimize the radar QPE statistically. In this study a variational approach is developed to quantitatively estimate the rainfall rate $(R)$ from the differential phase $\left(\Phi_{\mathrm{DP}}\right)$. A spline filter is utilized in the optimization procedures to eliminate the impact of the random errors in $\Phi_{\mathrm{DP}}$, which can be a major source of error in the specific differential phase $\left(K_{\mathrm{DP}}\right)$-based QPE. In addition, $R$ estimated from the horizontal reflectivity factor $\left(Z_{\mathrm{H}}\right)$ is used in the a priori with the error covariance matrix statistically determined. The approach is evaluated by an idealized case and multiple real rainfall cases observed by an operational S-band polarimetric radar in southern China. The comparative results demonstrate that with a proper range filter, the proposed variational radar QPE with the a priori included agrees well with the rain gauge measurements and proves to have better performance than the other three approaches, that is, the proposed variational approach without the a priori included, the variational approach proposed by Hogan, and the conventional power-law estimator-based approach.
\end{abstract}

\section{Introduction}

In combination with the conventional reflectivity factor $\left(Z_{\mathrm{H}}\right)$, polarimetric measurements, including the differential reflectivity $\left(Z_{\mathrm{DR}}\right)$, the differential phase/specific differential phase shift $\left(\Phi_{\mathrm{DP}} / K_{\mathrm{DP}}\right)$, and the copolar correlation coefficient $\left(\rho_{\mathrm{hv}}\right)$, provide information to improve the accuracy of radar quantitative precipitation estimation (QPE). Various QPE algorithms mostly in power-law form have been proposed to estimate rainfall rate $(R)$ from the polarimetric radar measurements, for example, $R\left(Z_{\mathrm{H}}\right), R\left(Z_{\mathrm{H}}, Z_{\mathrm{DR}}\right)$, $R\left(K_{\mathrm{DP}}\right), R\left(K_{\mathrm{DP}}, Z_{\mathrm{DR}}\right)$, and $R\left(A_{\mathrm{H}}\right)$ (Brandes et al. 2002 ; Bringi and Chandrasekar 2001; Marshall and Palmer 1948; Ryzhkov et al. 2005a, 2014, 2005b; Seliga and Bringi 1976). Different rainfall estimators show different error patterns that are related to the precipitation microphysics and the quality of polarimetric radar data (Lee 2006). The radar data quality can be attributed to various reasons, including radar

Corresponding author: Kun Zhao, zhaokun@nju.edu.cn; Guifu Zhang, guzhang1@ou.edu miscalibration, power attenuation, nonmeteorological target contamination, hail contamination, and random error (Berne and Krajewski 2013; Cifelli et al. 2011). To improve the accuracy of the rainfall estimation, different QPE algorithms are combined according to their error patterns or the precipitation classification, which are known as synthetic algorithms (Chen et al. 2017; Cifelli et al. 2011; Giangrande and Ryzhkov 2008; Ryzhkov et al. 2005b).

Among the polarimetric radar variables, $\Phi_{\mathrm{DP}}$, which is defined as the difference in the propagation phase shift of the horizontal and vertical polarized signals, and $K_{\mathrm{DP}}$, which is the range derivative of $\Phi_{\mathrm{DP}}$, are immune to radar miscalibration, attenuation, partial beam blockage, and contamination of solid-phase hydrometeors. QPE with $K_{\mathrm{DP}}$ is less affected by raindrop size distribution (DSD) variability than $\mathrm{QPE}$ using a $Z_{\mathrm{H}}-R$ relation, since $K_{\mathrm{DP}}$ is approximately the 4.2nd moment of DSDs (Huang et al. 2017) that is closer to the 3.67th moment of DSD (related to rain rate) than the 6 th moment $\left(Z_{\mathrm{H}}\right.$ for Rayleigh scattering). Unlike $Z_{\mathrm{H}}$ or $Z_{\mathrm{DR}}, K_{\mathrm{DP}}$ is not a direct radar measurement, so a straightforward way is to estimate $K_{\mathrm{DP}}$ 
from $\Phi_{\mathrm{DP}}$ before using it in QPE. A lot of methods have been proposed for $\Phi_{\mathrm{DP}}$ processing and $K_{\mathrm{DP}}$ estimation in the literature (Hubbert and Bringi 1995; Hubbert et al. 1993; Wang and Chandrasekar 2009). Random error and backscattering phase in the observed $\Phi_{\mathrm{DP}}$ could result in negative $K_{\mathrm{DP}}$ estimates that do not match the physical truth for rain measurements. To mitigate the impact of the abnormal $K_{\mathrm{DP}}$ values on QPE, Ryzhkov et al. (2005a) proposed to include the sign of $K_{\mathrm{DP}}$ in the estimator $\left[R=a\left|K_{\mathrm{DP}}\right|^{b} \operatorname{sign}\left(K_{\mathrm{DP}}\right)\right]$. However, including the sign could occasionally lead to negative accumulated rainfall. Recently, the linear programming method has been applied to $K_{\mathrm{DP}}$ estimation of rainfall (Giangrande et al. 2013, hereafter G13). Nonnegative $K_{\mathrm{DP}}$ constraints are imposed in the algorithms to reduce the impact of $\Phi_{\mathrm{DP}}$ errors and eventually lead to better QPE performance.

An alternative way for QPE with $\Phi_{\mathrm{DP}}$ is to use a forward approach (Rodgers 2000). In this method, $R$ is regarded as a state variable, and a forward model is built to predict $\Phi_{\mathrm{DP}}$ values from $R$. Generally, optimum $R$ estimates can be obtained when the difference between the predicted and the measured $\Phi_{\mathrm{DP}}$ is minimized. This method directly connects $R$ with $\Phi_{\mathrm{DP}}$ measurements, so the two-step error propagation from $\Phi_{\mathrm{DP}}$ to $K_{\mathrm{DP}}$ and then from $K_{\mathrm{DP}}$ to $R$ is avoided. Additional constraints, such as the nonnegativity of $R$ and the self-consistency of polarimetric variables, can be incorporated into the algorithm. The essential idea of this method is to optimally retrieve $R$ under the constraints posed by all the available information. The variational analysis is a common way to achieve this goal (Hogan 2007, hereafter H07). H07 proposed a variational QPE approach based on the $Z-R$ relation. The coefficient in the $Z-R$ relation is adjusted according to $Z_{\mathrm{DR}}$ and $\Phi_{\mathrm{DP}}$ measurements. In addition, the forward operator is built from idealized DSDs (gamma distribution with fixed $\mu$ ) in his work.

Based on the $R-K_{\mathrm{DP}}$ relation derived from the real DSD measurements, we develop a QPE approach with the variational approach acting as the optimization framework in this work. Section 2 gives the description of the variational QPE approach. An idealized case is utilized to verify the algorithm in section 3. In section 4 the radar measurements of multiple precipitation cases in southern China are utilized to quantitatively evaluate the algorithm's performance. Conclusions are given in section 5 .

\section{Description of the proposed variational QPE framework}
a. DSD dataset used to construct the variational $Q P E$ approach

As the variational QPE approach is designed for S-band polarimetric radars, and the evaluation is on the

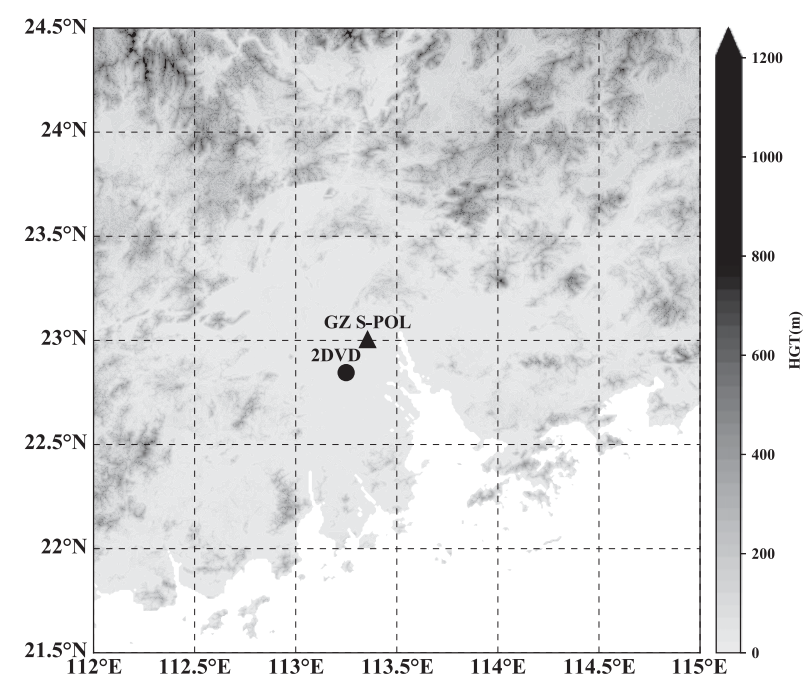

FIG. 1. The topography around Guangzhou S-POL (black triangle). The elevation of the terrain is indicated (shading). The 2DVD (black dot) is used to derive the statistical property of the precipitation in this region.

basis of the data collected by a polarimetric radar and rain gauges in southern China, we use the DSD data collected in the same region to find the statistical relations. A two-dimensional video disdrometer (2DVD), which is a highly accurate instrument for measuring precipitation particles (Schönhuber et al. 2007), deployed in Guangdong Province, China, collected DSD data from May to July in 2016 and 2017. The location of the 2DVD is shown in Fig. 1. To build the connection between the polarimetric variables $\left(Z_{\mathrm{H}}, Z_{\mathrm{DR}}\right.$, and $\left.K_{\mathrm{DP}}\right)$ and the rainfall rate, we calculate them from the DSD data following the formulas given by Cao et al. (2012). The scattering amplitudes are estimated with the T-matrix method (Mishchenko et al. 2000). The temperature is assumed to be $20^{\circ} \mathrm{C}$; the wavelength is $10.4 \mathrm{~cm}$. The statistical terminal velocity relation and the raindrop axis ratio relation are assumed to be the same as those given by Brandes et al. (2002).

\section{b. The basic variational analysis}

To estimate precipitation, the state vector $\mathbf{x}$ for an $n$-gate ray in a radar scan is defined as follows:

$$
\mathbf{x}=[R(1), R(2), \ldots, R(n)]^{\mathrm{T}},
$$

where $R(i)$ is rainfall rate at the $i$ th range gate and the superscript $\mathrm{T}$ means transpose. The $\Phi_{\mathrm{DP}}$ measurements can be denoted in vector form as follows:

$$
\mathbf{y}=\left[\Phi_{\mathrm{DP}}(1), \Phi_{\mathrm{DP}}(2), \ldots, \Phi_{\mathrm{DP}}(n)\right]^{\mathrm{T}},
$$

where $\mathbf{y}$ is called the observation vector and $\Phi_{\mathrm{DP}}(i)$ is the $\Phi_{\mathrm{DP}}$ measurement at the $i$ th range gate. The system 
phase and the folding in $\Phi_{\mathrm{DP}}$ measurements should be preprocessed beforehand. By assuming Gaussian-distributed $\Phi_{\mathrm{DP}}$ errors, the cost function $J$ of the variational analysis, which is sum of the cost functions for the a priori $\left(J_{b}\right)$ and the observations $\left(J_{\Phi_{\mathrm{DP}}}\right)$, can be defined as

$$
\begin{aligned}
J & =J_{b}+J_{\Phi_{\mathrm{DP}}}, \\
J_{b}(\mathbf{x}) & =\frac{1}{2}\left(\mathbf{x}-\mathbf{x}_{b}\right)^{\mathrm{T}} \mathbf{B}^{-1}\left(\mathbf{x}-\mathbf{x}_{b}\right), \\
J_{\Phi_{\mathrm{DP}}}(\mathbf{x}) & =\frac{1}{2} \delta \mathbf{y}^{\mathrm{T}} \mathbf{R}_{\Phi_{\mathrm{DP}}}^{-1} \delta \mathbf{y},
\end{aligned}
$$

where $\mathbf{x}_{b}$ and $\mathbf{B}$ are called the a priori and the corresponding error covariance matrix, respectively, which will be elaborated in section $2 \mathrm{~d} ; \delta \mathbf{y}$ is the innovation vector; and $\mathbf{R}_{\Phi_{\mathrm{DP}}}$ is the error covariance matrix of $\Phi_{\mathrm{DP}}$ measurements (Kalnay 2003), which is usually determined by radar signal processing. In this study the measurement errors of $\Phi_{\mathrm{DP}}$ are assumed to be independent at different range gates. As a consequence, the diagonal entries of $\mathbf{R}_{\Phi_{\mathrm{DP}}}$ are set to the standard deviation of the $\Phi_{\mathrm{DP}}$ estimation errors and the offdiagonal elements are set to zero. The innovation vector $\delta \mathbf{y}$, defined as $\delta \mathbf{y}=H(\mathbf{x})-\mathbf{y}$, is the difference between $\mathbf{y}$ and the vector of propagation differential phase $\left(\phi_{\mathrm{DP}}\right)$ predicted from the rainfall rate $\mathbf{x}$, and $H(\cdot)$ is called the forward operator mapping the state variables to the measurements (Rodgers 2000). In this study the fitted powerlaw relation between $R$ and $K_{\mathrm{DP}}$-that is, $K_{\mathrm{DP}}=a R^{b}$ $\left(a=5.767 \times 10^{-3}, b=1.274\right)$, which corresponds to $R=57.132 K_{\mathrm{DP}}^{0.785}$ shown in Fig. 2-is utilized to convert $\mathbf{x}$ to the $K_{\mathrm{DP}}$ vector. Then, the predicted $\phi_{\mathrm{DP}}$ at $j$ th range gate can be obtained with the accumulation, as follows:

$$
\begin{aligned}
\phi_{\mathrm{DP}}(j) & =2 \delta r \sum_{i=1}^{j-1} K_{\mathrm{DP}}(i), \\
& =2 a \delta r \sum_{i=1}^{j-1} R(i)^{b},
\end{aligned}
$$

where $K_{\mathrm{DP}}(i)$ is the predicted $K_{\mathrm{DP}}$ at the $i$ th range gate and $\delta r$ is the range gate resolution.

The statistically optimum $R$ for all the range gates is the state vector $\mathbf{x}$ when Eq. (3) is minimized. In fact, the forward operator is not linear $(b \neq 1)$; the minimization is actually a nonlinear optimization problem as a consequence. A common method for simplifying the optimization is to linearize the forward operator, converting Eq. (3) to a quadratic function, and then to minimize the cost function iteratively. This method is usually applied on the data assimilation of numerical weather prediction (Kalnay 2003). The estimate of the state vector at the iteration $(k-1)$ is denoted as $\mathbf{x}_{k-1}$. Then, the innovation vector at the $k$ th iteration can be linearized as

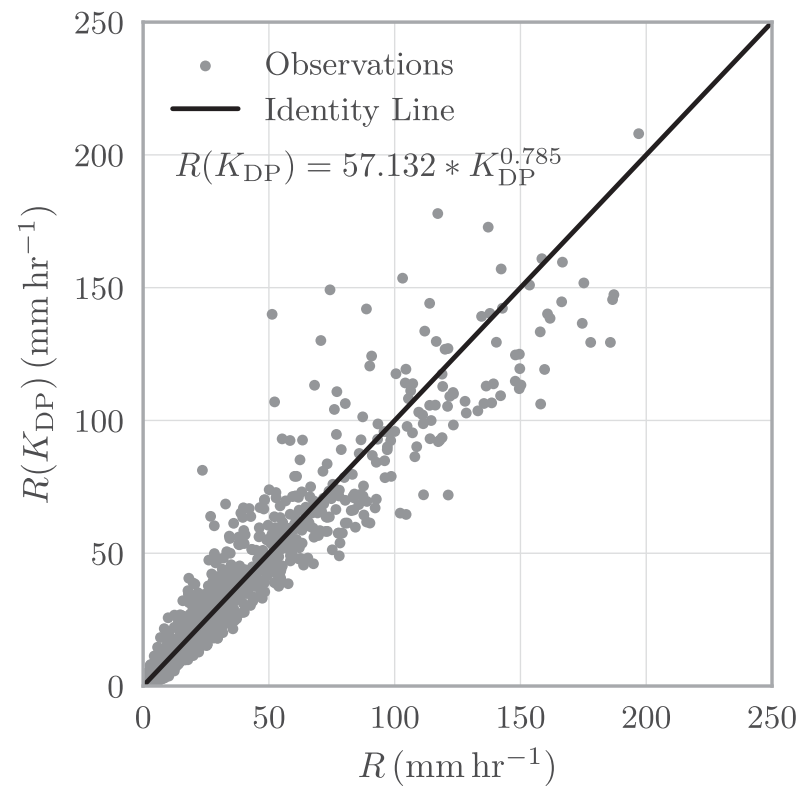

FIG. 2. The statistical QPE relations $R\left(K_{\mathrm{DP}}\right)$ for S-band radars obtained from the regression of the observations (gray dots).

$$
\begin{aligned}
\delta \mathbf{y} & =H(\mathbf{x})-\mathbf{y} \\
& \approx \mathbf{H} \cdot\left(\mathbf{x}-\mathbf{x}_{k-1}\right)+H\left(\mathbf{x}_{k-1}\right)-\mathbf{y},
\end{aligned}
$$

where $\mathbf{H}$ is the Jacobian matrix containing the partial derivative of the predicted observation $H\left(\mathbf{x}_{k-1}\right)$ with respect to each element of $\mathbf{x}_{k-1}$, expressed as

$$
\frac{\partial \phi_{\mathrm{DP}}(j)}{\partial R(i)}= \begin{cases}0 & j \leq i \\ 2 a b \delta r R(i)^{b-1} & j>i\end{cases}
$$

and

$$
\begin{gathered}
\mathbf{H}=2 \delta r\left[\begin{array}{cccc}
\frac{\partial \phi_{\mathrm{DP}}(1)}{\partial R(1)} & \frac{\partial \phi_{\mathrm{DP}}(1)}{\partial R(2)} & \ldots & \frac{\partial \phi_{\mathrm{DP}}(1)}{\partial R(n)} \\
\frac{\partial \phi_{\mathrm{DP}}(2)}{\partial R(1)} & \frac{\partial \phi_{\mathrm{DP}}(2)}{\partial R(2)} & \ldots & \frac{\partial \phi_{\mathrm{DP}}(2)}{\partial R(n)} \\
\vdots & \vdots & \ddots & \vdots \\
\frac{\partial \phi_{\mathrm{DP}}(n)}{\partial R(1)} & \frac{\partial \phi_{\mathrm{DP}}(n)}{\partial R(2)} & \ldots & \frac{\partial \phi_{\mathrm{DP}}(n)}{\partial R(n)}
\end{array}\right] \\
=2 a b \delta r\left[\begin{array}{ccccc}
0 & & & \\
R(1)^{b-1} & 0 & & \mathbf{O} \\
\vdots & \vdots & \ddots & \\
R(1)^{b-1} & R(2)^{b-1} & \ldots & 0 \\
R(1)^{b-1} & R(2)^{b-1} & \ldots & R(n-1)^{b-1} & 0
\end{array}\right],
\end{gathered}
$$


where $R(i)$ is the elements of $\mathbf{x}_{k-1} ; \mathbf{H}$ is a lower triangular matrix; and the entries of its main diagonal are also zero.

The linearized cost function and the corresponding gradient at the $k$ th iteration are given by

$$
\begin{aligned}
J_{k}= & \frac{1}{2}\left(\mathbf{x}_{k}-\mathbf{x}_{b}\right)^{\mathrm{T}} \mathbf{B}^{-1}\left(\mathbf{x}_{k}-\mathbf{x}_{b}\right) \\
& +\frac{1}{2}\left[\mathbf{H} \cdot\left(\mathbf{x}_{k}-\mathbf{x}_{k-1}\right)+H\left(\mathbf{x}_{k-1}\right)-\mathbf{y}\right]^{\mathrm{T}} \mathbf{R}_{\Phi_{\mathrm{DP}}}^{-1} \\
& \times\left[\mathbf{H} \cdot\left(\mathbf{x}_{k}-\mathbf{x}_{k-1}\right)+H\left(\mathbf{x}_{k-1}\right)-\mathbf{y}\right],
\end{aligned}
$$

and

$$
\begin{aligned}
\mathbf{g}\left(\mathbf{x}_{k}\right)= & \nabla_{\mathbf{x}_{k}} J_{k} \\
= & \mathbf{B}^{-1}\left(\mathbf{x}_{k}-\mathbf{x}_{\mathrm{b}}\right) \\
& +\mathbf{H}^{\mathrm{T}} \mathbf{R}_{\Phi_{\mathrm{DP}}}^{-1}\left[\mathbf{H} \cdot\left(\mathbf{x}_{k}-\mathbf{x}_{k-1}\right)+H\left(\mathbf{x}_{k-1}\right)-\mathbf{y}\right],
\end{aligned}
$$

where $\mathbf{g}\left(\mathbf{x}_{k}\right)$ is the gradient of $J_{k}$ with respect to $\mathrm{x}_{k}$, and $\nabla$ represents the gradient. With the cost function, the corresponding gradient, and the constraints for the state variables (nonnegative rainfall rate), the problem can be iteratively solved using the constrained optimization algorithms (Sun and Yuan 2006), in which the Hessian of the cost function is approximately estimated automatically (Byrd et al. 1995). The optimization starts from a first guess, that is, $\mathbf{x}_{0}=\mathbf{x}_{g}$, which can be a statistical state, the a priori, or the solution of the previous ray of the radar scan. In our study the a priori is utilized, that is, $\mathbf{x}_{g}=\mathbf{x}_{b}$. Once the linearized cost function is minimized, the solution is utilized as the guess for the next iteration. Once the solution is converged satisfactorily, it is the statistically optimum estimate. A $\chi^{2}$ convergence test can be used here.

\section{c. Range smoothing with the cubic B spline}

The smoothing filter plays an important role in the variational approach. The forward model [see Eq. (4)] has a cumulative-sum form, and the state variable in a single range gate provides less information than the random errors. The rainfall rate will be overfitted to the errors if no additional constraint is introduced. Fortunately, the precipitation in nature measured by radars tends to be spatially continuous, which can be ensured by a smoothing mechanism in the variational analysis. Commonly, there are three ways to smooth the state vector in the minimization procedures of the cost function. The first is to introduce a smoothing filter for the state vector; the second is to add a penalty term, which is commonly a mean square of a Laplacian of the state vector or any other term with a similar function, to the cost function to impose additional smoothing constraints of the state vector; the third way is to use a nondiagonal a priori covariance matrix B (H07; Maesaka et al. 2012).

In our study the cubic basis spline (B-spline) similar to that used by $\mathrm{H} 07$ is adopted. A low-resolution state vector, denoted as $\hat{\mathbf{x}}=[x(1), x(2), \ldots, x(m)]^{\mathrm{T}}$, is introduced. It is converted to the rainfall-rate estimates at the radar range gates ( $\mathbf{x}$ ) with an $n \times m$ matrix $\mathbf{W}$ consisting of the weights of the cubic B-spline,

$$
\mathbf{x}=\mathbf{W} \hat{\mathbf{x}} .
$$

Matrix $\mathbf{W}$ is essentially an interpolation operator, and it constructs the high-resolution $\mathbf{x}$ from the interpolation of $\hat{\mathbf{x}}$. The smoothness of $\mathbf{x}$ is mainly controlled by the smoothing factor $c(m \approx n / c)$. The details of matrix $\mathbf{W}$ can be found in the appendix of H07's study. With the matrix $\mathbf{W}$ included, the cost function and the corresponding gradient become

$$
\begin{aligned}
J_{k}= & \frac{1}{2}\left(\mathbf{W} \hat{\mathbf{x}}_{k}-\mathbf{x}_{b}\right)^{\mathrm{T}} \mathbf{B}^{-1}\left(\mathbf{W} \hat{\mathbf{x}}_{k}-\mathbf{x}_{b}\right) \\
& +\frac{1}{2}\left[\mathbf{H} \cdot\left(\mathbf{W} \hat{\mathbf{x}}_{k}-\mathbf{W} \hat{\mathbf{x}}_{k-1}\right)+H\left(\mathbf{W} \hat{\mathbf{x}}_{k-1}\right)-\mathbf{y}\right]^{\mathrm{T}} \mathbf{R}_{\Phi_{\mathrm{DP}}}^{-1} \\
& \cdot\left[\mathbf{H} \cdot\left(\mathbf{W} \hat{\mathbf{x}}_{k}-\mathbf{W} \hat{\mathbf{x}}_{k-1}\right)+H\left(\mathbf{W} \hat{\mathbf{x}}_{k-1}\right)-\mathbf{y}\right]
\end{aligned}
$$

and

$$
\begin{aligned}
\mathbf{g}\left(\hat{\mathbf{x}}_{k}\right)= & \nabla_{\hat{\mathbf{x}}_{k}} J_{k} \\
= & \mathbf{W}^{\mathrm{T}} \mathbf{B}^{-1}\left(\mathbf{W} \hat{\mathbf{x}}_{k}-\mathbf{x}_{b}\right) \\
& +\mathbf{W}^{\mathrm{T}} \mathbf{H}^{\mathrm{T}} \mathbf{R}_{\Phi_{\mathrm{DP}}}^{-1}\left[\mathbf{H} \cdot\left(\mathbf{W} \hat{\mathbf{x}}_{k}-\mathbf{W} \hat{\mathbf{x}}_{k-1}\right)+H\left(\mathbf{W} \hat{\mathbf{x}}_{k-1}\right)-\mathbf{y}\right] .
\end{aligned}
$$

Now the variable to be optimized is $\hat{\mathbf{x}}$, and once convergence is achieved, $\mathbf{W} \hat{\mathbf{x}}$ is the estimated rainfall rates at the radar range gates. And the intermediate variables-for example, $\phi_{\mathrm{DP}}$ and $K_{\mathrm{DP}}$-can be estimated from rainfall rates using the forward operator.

\section{d. The a priori and the error covariance matrix}

The a priori and the error covariance matrix are the information we know before the estimation and its uncertainty. If we use no additional information for QPE, then the diagonal entries of the a priori error covariance can be set to infinity. As a consequence only $\Phi_{\mathrm{DP}}$ is used in the variational analysis. In such a case, the approach is similar to the conventional $R\left(K_{\mathrm{DP}}\right)$ method with the additional physical constraints included (G13; Huang et al. 2017). As we know, when the rainfall is light, the useful information provided by $\Phi_{\mathrm{DP}}$ measurements is contaminated by the errors in $\Phi_{\mathrm{DP}}$. In the 

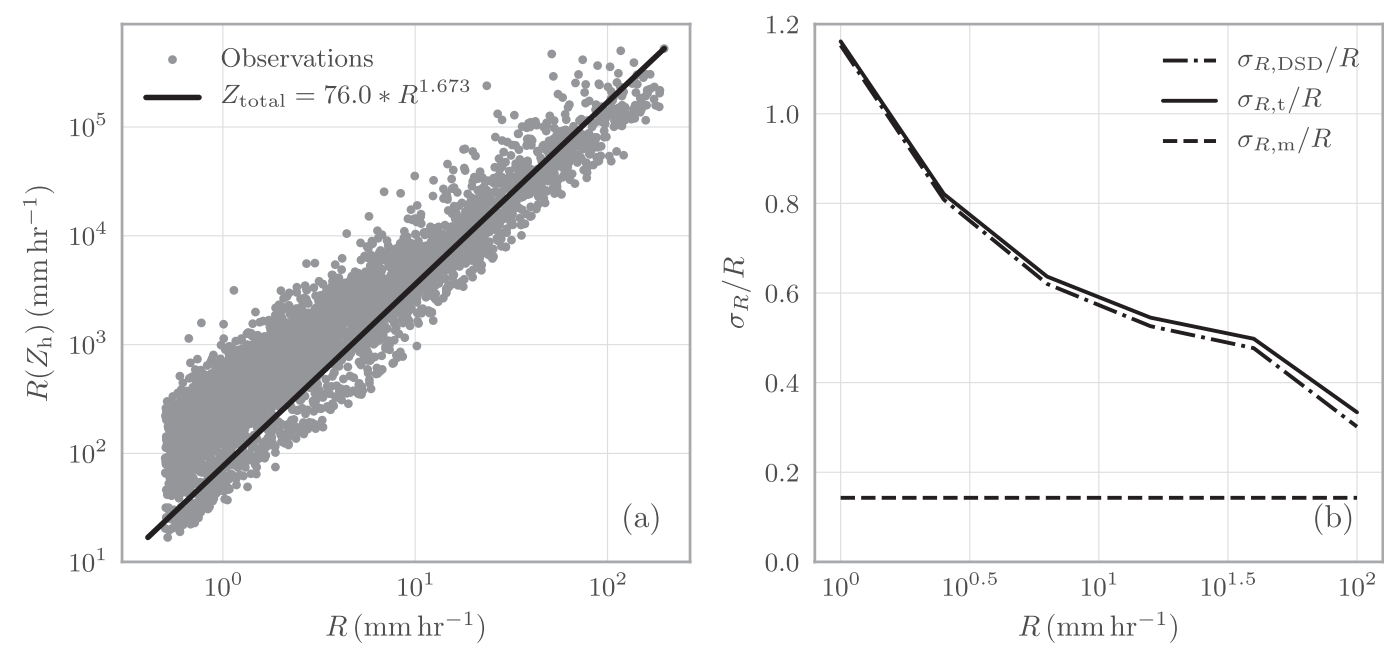

FIG. 3. (a) The statistical $R-Z_{\mathrm{H}}$ relation (black solid line) for S-band radars regressed from the observations (gray dots). (b) The normalized standard deviation in $R$ for the $Z_{\mathrm{H}}-R$ relation caused by DSD variability $\left(\sigma_{R, \mathrm{DSD}} / R\right.$; black dashed-dotted line) and measurement noise $\left(\sigma_{R, m} / R\right.$; black dashed line $)$. The total error $\left(\sigma_{R, t} / R\right.$; black solid line $)$, which is a combination of $\sigma_{R, \mathrm{DSD}} / R$ and $\sigma_{R, m} / R$.

synthetic rainfall algorithms (Bringi et al. 2011, 2009; Cifelli et al. 2011), the $Z_{\mathrm{H}}$ or $R\left(Z_{\mathrm{H}}\right)$ is used as the threshold conditions for selecting the QPE algorithms. For example, $R\left(Z_{\mathrm{H}}\right)$ is usually used for light rain because the rainfall estimated from $Z_{\mathrm{DR}}$ or $K_{\mathrm{DP}}$ has a relatively large error. Inspired by this idea, we incorporate $R\left(Z_{\mathrm{H}}\right)$ from the statistical $Z-R$ relation into the variational analysis as the a priori term to provide more useful information in the variational analysis. Note that this is different from the conventional a priori term that usually uses a fixed value estimated from the climatic statistics, but we still call it the a priori because the adopted statistical $Z-R$ relation can represent the mean characteristics of the DSDs in the regime. The a priori error covariance matrix $\mathbf{B}$ is utilized to characterize the error properties of the a priori. The diagonal entries of $\mathbf{B}$ (denoted as $\sigma_{R \text {,ap }}$ ) describe the statistical standard deviation of the error of the a priori at each range gate, which consists of two parts, that is, the impact of the DSD variability and the measurement error of $Z_{\mathrm{H}}$ (Lee 2006), and the off-diagonal part is the joint variability of the a priori at the range gates.

The main focus in this study is the QPE of the S-band polarimetric radars in southern China. In our study the statistical relation $R_{\text {ap }}=0.0752 Z_{\mathrm{h}}^{0.598}$ (see Fig. 3a) is utilized as the a priori for both the idealized experiment and the real cases. Meanwhile, the error covariance is also calculated statistically using the dataset elaborated on in section 2a. The $\sigma_{R \text {,ap }}$ is attributed to the DSD variability and the measurement errors of $Z_{\mathrm{H}}$. The uncertainty resulting from the DSD variability $\left(\sigma_{R, \mathrm{DSD}} / R\right)$ can be derived as a function of rainfall rate, as shown by the dashed-dotted line in Fig. 3b. The uncertainty caused by the measurement noise $\left(\sigma_{R, m} / R\right)$ for the power-law relation is related to the measurement error of $Z_{\mathrm{H}}$ (Bringi and Chandrasekar 2001), and is given as

$$
\frac{\sigma_{R, m}}{R}=0.598\left(\frac{\sigma_{Z_{\mathrm{h}}}}{Z_{\mathrm{h}}}\right)
$$

where $\sigma_{Z_{\mathrm{h}}}$ represents the standard deviation of the linearized horizontal reflectivity factor $\left(Z_{\mathrm{h}}=10^{0.1 Z_{\mathrm{H}}}\right)$. If the error of the measurement noise of $Z_{\mathrm{H}}$ is about $1 \mathrm{~dB}$, then $\sigma_{R, m} / R$ is about $14 \%$ for our $Z_{\mathrm{H}}-R$ relation, and total uncertainty $\left(\sigma_{R, t} / R\right)$ is the collective effect of $\sigma_{R, \mathrm{DSD}} / R$ and $\sigma_{R, m} / R$ (Lee 2006), which yields

$$
\sigma_{R, t} / R=\sqrt{\left(\sigma_{R, \mathrm{DSD}} / R\right)^{2}+\left(\sigma_{R, m} / R\right)^{2}} .
$$

We denote the uncertainty $\left(\sigma_{R, t} / R\right)$ as a function of the rainfall rate $f(R)$. Thus, in the variational analysis, the diagonal entries of the a priori error covariance matrix $\mathbf{B}$ is estimated from the a priori, using $\sigma_{R \text {,ap }}=R_{\text {ap }} \times f\left(R_{\text {ap }}\right)$. Since the $Z_{\mathrm{H}}$ measurements are independent at the different range gates, the off-diagonal parts of the a priori error covariance are set to zero. The $\sigma_{R \text {,ap }}$ is set to $2 \mathrm{~mm} \mathrm{~h}^{-1}$ if the estimated value is less. In real applications, hail probably exists at the range gates where $Z_{\mathrm{H}}$ values are greater than $53 \mathrm{~dB} Z$. Then, the corresponding $\sigma_{R \text {,ap }}$ values are set to infinity.

For radars of short wavelength, such as $\mathrm{C}$ or $\mathrm{X}$ band, the backscattering phase in $\Phi_{\mathrm{DP}}$ measurements and propagation path attenuation are major obstacles for variational QPE. In this situation both $\Phi_{\mathrm{DP}}$ and $Z_{\mathrm{H}}$ provide less useful information. Then, the attenuation correction of $Z_{\mathrm{H}}$ is necessary for the a priori (Carey et al. 2000), and the attenuation effect should also be included in the error covariance, which is left for future research. 

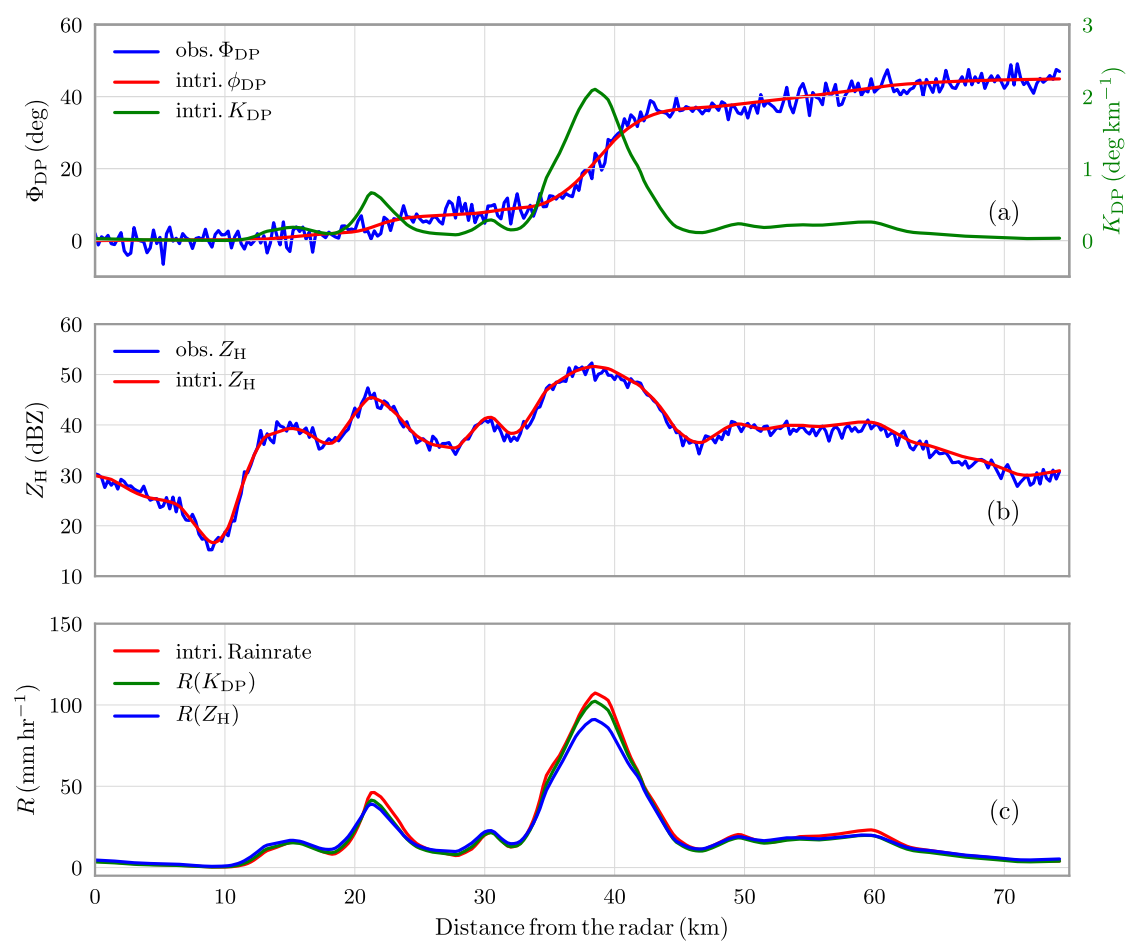

FIG. 4. The range profiles of the polarimetric variables and the rainfall rate used in the idealized case. (a) The intrinsic $\phi_{\mathrm{DP}}$ (red line) and the simulated $\Phi_{\mathrm{DP}}$ (blue line) measurements, and the intrinsic $K_{\mathrm{DP}}$ (green line). (b) The intrinsic $Z_{\mathrm{H}}$ (red line) and the simulated $Z_{\mathrm{H}}$ (blue line) measurements. (c) The intrinsic rainfall rate (red line), and the rainfall rates estimated from the statistical $R-K_{\mathrm{DP}}$ relation (green line) and the $Z_{\mathrm{H}^{-}}-R$ relation (blue line).

In this study we focus on QPE of the operational S-band radar in southern China.

\section{Verification with an idealized case}

\section{a. Experiment design}

In a sense the temporal evolution of rain properties is similar to the spatial variability, since the weather systems are moving. Thus, we simulate a range profile of radar measurements to test our QPE method based on the 2DVD observations. A rainfall event passed over the 2DVD site from 0830 to 1100 UTC 28 June 2016, in which the maximum 1-min rainfall rate exceeded $100 \mathrm{~mm} \mathrm{~h}^{-1}$. Since the 2DVD has a small sampling volume, the short-term rain properties can be significantly different from the climatic statistics as a result of the internal microscale variability of precipitation systems. Thus, a Bayesian method similar to that developed by Cao et al. (2010) is employed so that the simulation is closer to the statistics. First, the series of synthetic $Z_{\mathrm{H}}$ and $Z_{\mathrm{DR}}$ are calculated from the 2DVD data based on the scattering amplitudes used earlier. DSD parameters $N_{0}, \mu$, and $\Lambda$ are calculated from the synthetic $Z_{\mathrm{H}}$ and $Z_{\mathrm{DR}}$ series using the Bayesian method based on the $\mu-\Lambda$ relation derived from the 2-yr summertime DSD observations $\left(\mu=-0.01510 \Lambda^{2}+1.033 \Lambda-2.042\right)$. The range resolution of the profiles is assumed to be $250 \mathrm{~m}$. Considering that the storm moved more than $250 \mathrm{~m}$ in $1 \mathrm{~min}$ and our goal is to create simulated truth to test the retrieval method, the DSD parameters $N_{0}, \mu$, and $\Lambda$ are interpolated and filtered with the five-gate moving median and mean filters to make the storm scale more realistic. Then, the range profiles of $R, Z_{\mathrm{H}}$, and $K_{\mathrm{DP}}$ can be calculated from $N_{0}, \mu$, and $\Lambda$ with the assumption of the gamma distribution. The $\Phi_{\mathrm{DP}}$ range profile is obtained from the integral of $K_{\mathrm{DP}}$ following Eq. (4). To simulate the observations, unbiased Gaussian random errors should be added to $Z_{\mathrm{H}}$ and $\Phi_{\mathrm{DP}}$. The attenuation effect and the backscattering phase are neglected for this S-band experiment. Figure 4 shows an example of the simulated $Z_{\mathrm{H}}$ and $\Phi_{\mathrm{DP}}$ observations. The standard deviations of simulated errors in $Z_{\mathrm{H}}$ and $\Phi_{\mathrm{DP}}$ are $1 \mathrm{~dB}$ and $2^{\circ}$, respectively. The range profiles of the intrinsic $R$, $R\left(Z_{\mathrm{H}}\right)$, and $R\left(K_{\mathrm{DP}}\right)$ are shown in Fig. 4c. The heavy precipitation center is located about $38 \mathrm{~km}$ away from the radar. The rainfall rates estimated from $Z_{\mathrm{H}}$ and $K_{\mathrm{DP}}$ are not exactly same with the intrinsic $R$ because of DSD variability. As a consequence, the uncertainty in the forward operator is included in the idealized case. 

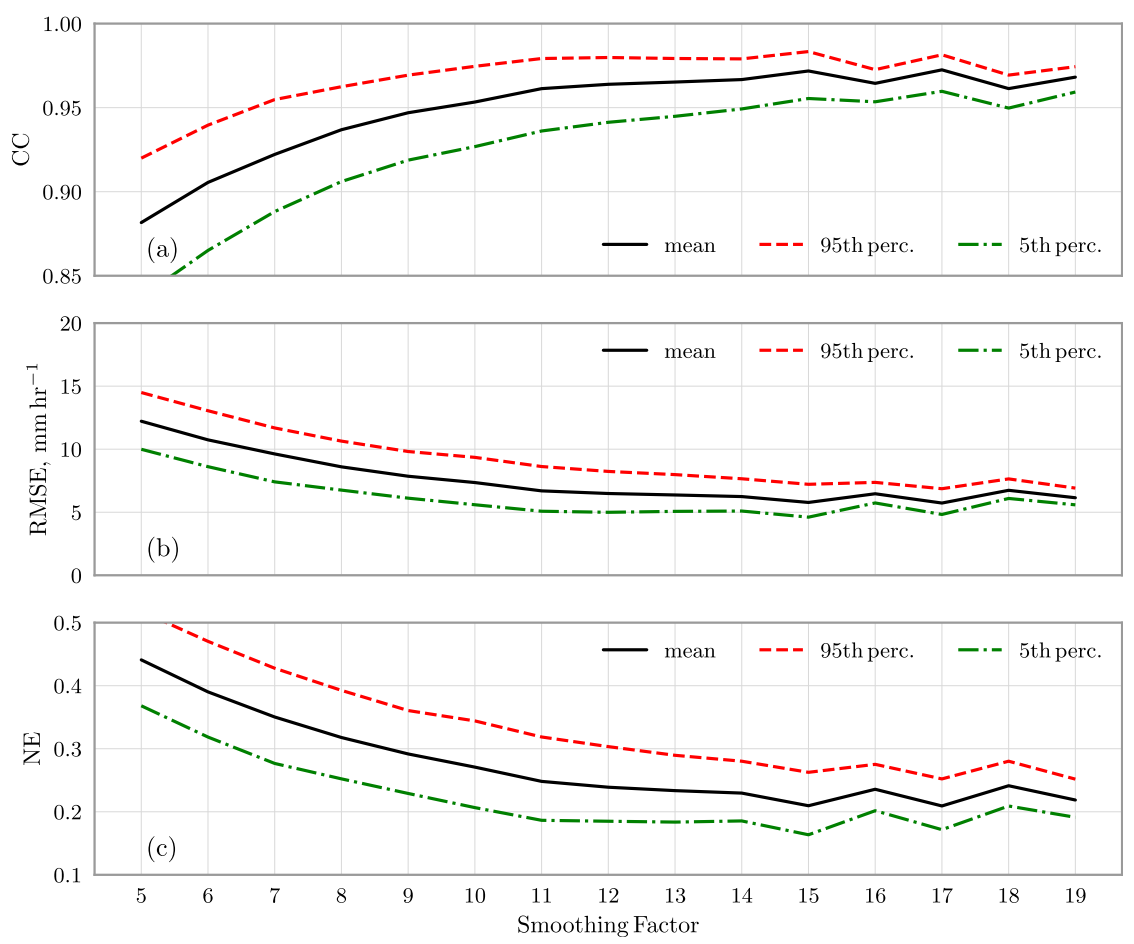

FIG. 5. The statistics of the (a) CCs, (b) RMSEs, and (c) NEs corresponding to the 1000 range profiles of $R$ estimated with the variational approach using different smoothing factors. The mean (black solid line), the 95th percentile (red dashed line), and the 5th percentile (green dashed-dotted line) of the statistical variables are marked.

In later subsections three tests are conducted to show the performance of the variational QPE approach. First, estimates of $R$ using a different smoothing factor are intercompared so that a proper smoothing factor is decided for the idealized case and also the real application. Then, the performance of the variational QPE approach is compared with that of G13's method. At last, the impact of the a priori statistics on the $R$ estimates is investigated. To quantitatively evaluate the estimation, the correlation coefficient (CC), the root-mean-square error (RMSE), and the normalized absolute error (NE) are utilized, which are defined as

$$
\begin{aligned}
\mathrm{CC} & =\frac{\sum_{i=1}^{N}\left(R_{s, i}-\overline{R_{\mathrm{s}}}\right)\left(R_{e, i}-\overline{R_{e}}\right)}{\sqrt{\sum_{i=1}^{N}\left(R_{s, i}-\overline{R_{s}}\right)} \sqrt{\sum_{i=1}^{N}\left(R_{e, i}-\overline{R_{e}}\right)}} \\
\mathrm{RMSE} & =\left[\frac{1}{N} \sum_{i=1}^{N}\left(R_{s, i}-R_{e, i}\right)^{2}\right]^{\frac{1}{2}} \\
\mathrm{NE} & =\frac{\frac{1}{N} \sum_{i=1}^{N}\left|R_{s, i}-R_{e, i}\right|}{\overline{R_{s}}} .
\end{aligned}
$$

Here, $R_{s, i}$ is the standard rainfall rate at the $i$ th range gate, which represents the intrinsic values; $R_{e, i}$ is the rainfall-rate estimate at the $i$ th range gate; $\overline{R_{s}}$ and $\overline{R_{e}}$ are the corresponding expected values; and $N$ represents the total number of range gates.

\section{b. Impact of the smoothing filter}

As stated earlier, the smoothness of the estimates is decided by $c$. A larger smoothing factor results in smoother estimates, but it will also suppress the physical variation of the precipitation. A proper smoothing filter, which keeps the continuity of the rainfall while retaining as much detail as possible, is one of the keys to highperformance QPE. To find a proper smoothing factor, we use a statistical method. Based on the random-generated Gaussian measurement errors, 1000 range profiles of the simulated $Z_{\mathrm{H}}$ and $\Phi_{\mathrm{DP}}$ are generated from the simulated rainfall-rate series. The standard deviations of the simulated $Z_{\mathrm{H}}$ and $\Phi_{\mathrm{DP}}$ errors in each range profile are assumed to be equal to $1 \mathrm{~dB}$ and $2^{\circ}$, respectively. In the variational analysis, the $\Phi_{\mathrm{DP}}$ errors used in Eq. (11) are assumed equal to $2^{\circ}$, with no error correlation assumed. The diagonal entries of the a priori error covariance matrix are set close to infinity $\left(10^{20} \mathrm{~mm}^{2} \mathrm{~h}^{-2}\right)$, so the a priori provides almost no information here. The impact of the a priori will 

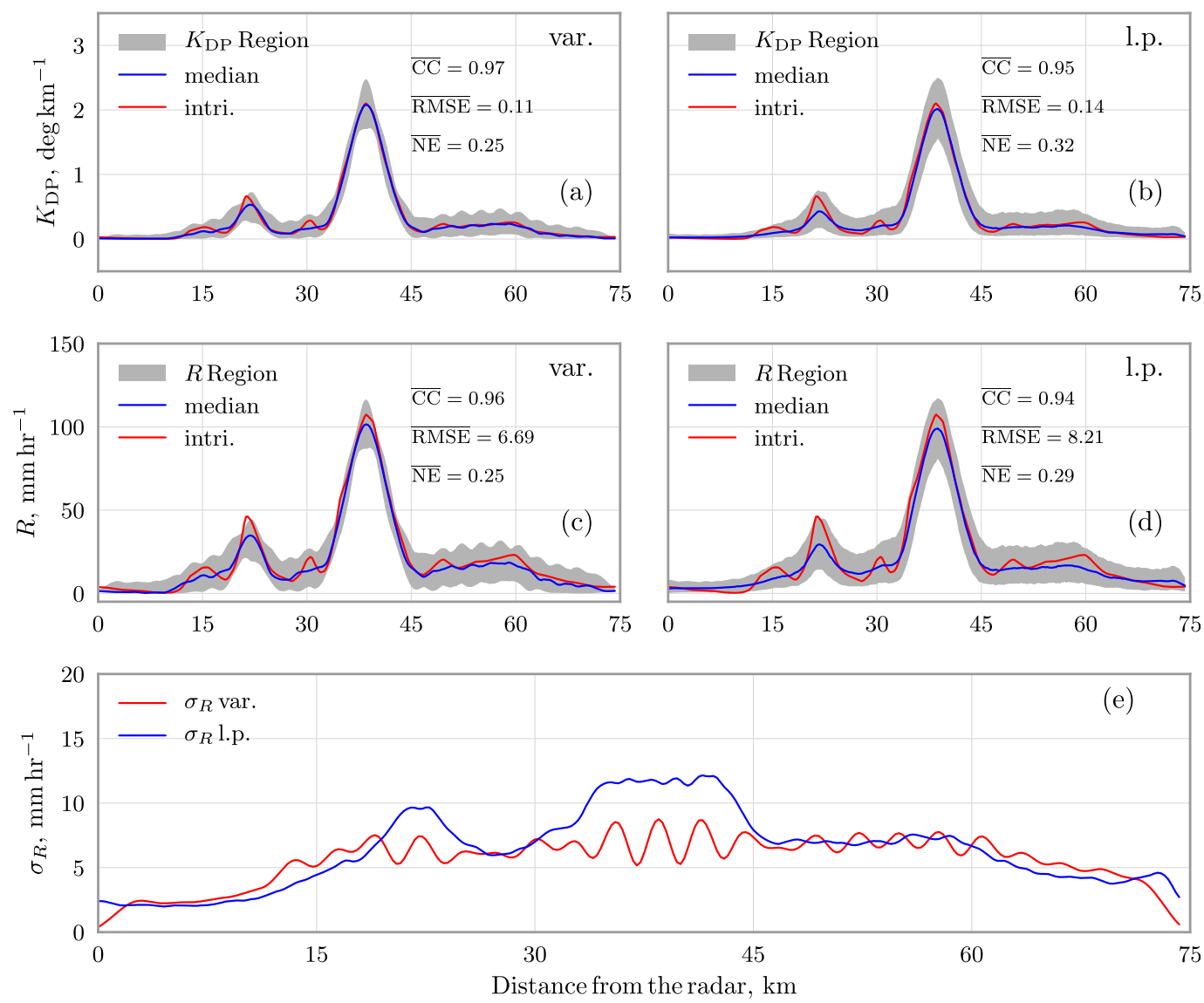

FIG. 6. (a)-(d) The statistical comparisons of the $R$ estimates and $K_{\mathrm{DP}}$ estimates using the variational approach, and G13's method based on 1000 range profiles of $Z_{\mathrm{H}}$ and $\Phi_{\mathrm{DP}}$ with random errors. No a priori information is utilized in the variational estimation here. (a) Estimates of $K_{\mathrm{DP}}$ based on the variational approach, (b) $K_{\mathrm{DP}}$ estimates based on G13's method, (c) $R$ estimates based on the variational approach, and (d) $R$ estimates based on G13's method. The median values of the estimates are indicated (blue solid lines). The regions between the 5th and 95th percentiles are marked (gray shading). The intrinsic $R$ and $K_{\mathrm{DP}}$ are indicated (red solid lines). (e) The comparison of the standard errors in the $R$ estimates using the variational approach (red solid line) and G13's method (blue solid line).

be studied in section $3 \mathrm{~d}$. The smoothing factor $c(m \approx n / c)$ is changed from 5 to 22 . Then, the statistics of the CCs, RMSEs, and NEs corresponding to the 1000 estimated range profiles of $R$ are utilized to represent the performance of the variational approach using a specific smoothing factor, as shown in Fig. 5.

With a smaller smoothing factor (less than 10), the mean $\mathrm{CC}$ is relatively lower and the mean RMSE/NE is relatively higher because of the impact of the residual low-frequency part of the random errors. When the smoothing factor increases, the impact of the random errors is weakened, the mean CC gets higher, and the mean RMSE/NE becomes lower at first, and then the mean CC decreases and the mean RMSE/NE increases again because of the excessive smoothing of the physical variation of rain. The performance of the variational approach achieves the best result when the weakening of the random errors and the smoothing of the physical variation generally balance each other. Overall, by using the smoothing factor between 11 and 15, the mean CC obtains the highest level and the mean RMSE/NE reaches the lowest level, indicating a satisfactory performance. To reduce the effects of the random errors while keeping the physical variation as much as possible, the smoothing factor is set to 11 in this study.

\section{c. Comparisons with $K_{D P}$ estimates based on linear programming}

The variational QPE approach is known as an optimization using the forward model based on $\Phi_{\mathrm{DP}}$ measurements. In contrast, the conventional QPE involves the inverse method, which estimates $K_{\mathrm{DP}}$ from $\Phi_{\mathrm{DP}}$ first and then calculates $R$ using the $R-K_{\mathrm{DP}}$ relations. G13 proposed to incorporate the nonnegativity constraint when estimating $K_{\mathrm{DP}}$ from $\Phi_{\mathrm{DP}}$ based on the linear programming, which proves to perform well. To investigate the 

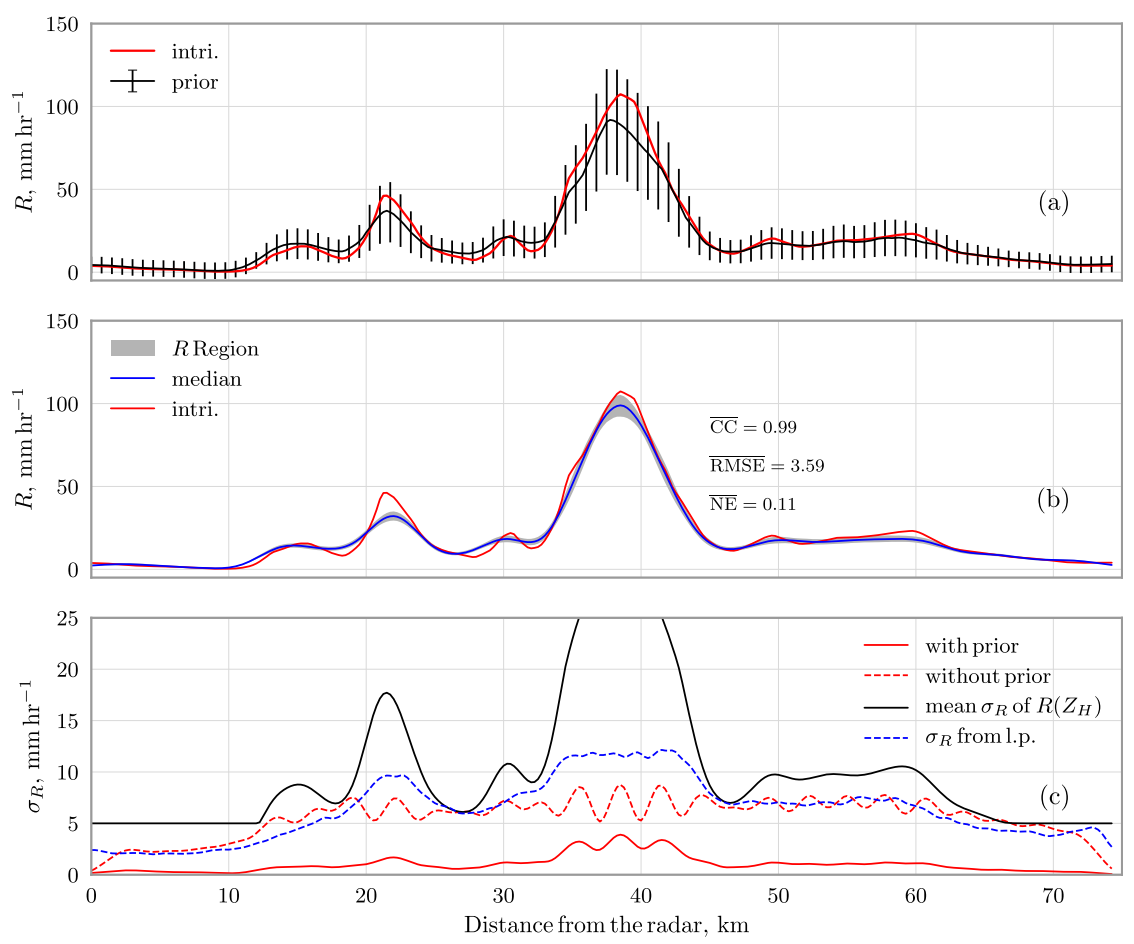

FIG. 7. (a) An example of the a priori (black solid line) utilized in the optimization and its corresponding errors (error bars). The intrinsic $R$ is shown (red solid line as a reference). (b) As in Fig. 6c, but for the $R$ estimates with the a priori used in the variational approach. (c) The comparison of the errors (standard deviations) in the $R$ estimates with the a priori used in the variational approach (red solid line), the $R$ estimates with no a priori used in the variational approach (red dashed line), and the $R$ estimates based on G13's method (blue dashed line). The mean standard deviation of the errors of the a priori is also shown (black solid line).

performance of the variational QPE approach, we compare its statistical results with those based on G13's method.

The comparisons are shown in Fig. 6, which is also based on 1000 range profiles of $Z_{\mathrm{H}}$ and $\Phi_{\mathrm{DP}}$ with random errors. The configuration of the variational QPE approach is the same as that used in section $3 \mathrm{~b}$, except that we use 11 as the smoothing factor $(m \approx n / 11)$ here. For G13's linear programming method, a nine-point derivative filter is utilized, which proves to be the best configuration for the idealized case (the procedure is not shown). Overall, uncertainty exists in the optimized $\phi_{\mathrm{DP}}$, the optimized $K_{\mathrm{DP}}$, and the estimated $R$ from both algorithms as a result of the random errors in the simulated observations. The optimized $\phi_{\mathrm{DP}}$ is highly consistent with the intrinsic value, with the mean CC close to 1 , the mean RMSE less than $0.30^{\circ}$, and the mean NE less than 0.01 for both methods (not shown). For the $K_{\mathrm{DP}}$ estimation, both methods give satisfactory results, with the mean values of $\mathrm{CC}$ greater than 0.96 and relatively low mean RMSE/NE. The median values of the $K_{\mathrm{DP}}$ estimates (denoted with the blue solid line) are generally close to the intrinsic values (denoted with the red solid line) as shown in Figs. 6a and 6b. For the
$R$ estimation, a little bit of inaccuracy exists because of the uncertainty in the forward operator (the $R-K_{\mathrm{DP}}$ relation). From the statistical perspective, the results from the variational approach are slightly better than those from G13's method. The median values from the variational approach also coincide better with the intrinsic values. Note that there is a slight underestimation in $R$ and $K_{\mathrm{DP}}$ from G13's method at the most severe part of the echo (intrinsic $R$ greater than $100 \mathrm{~mm} \mathrm{~h}^{-1}$ ). This is because the derivative filter used is a bit too large. With a longer derivative filter, the underestimation becomes more evident. The uncertainty in the $R$ estimates $\left(\sigma_{R}\right)$ caused by the random errors is generally comparable for the two methods. Within the range of $0 \sim 15 \mathrm{~km}$, where the intrinsic $R$ values are low, the variance in the $R$ estimates is relatively small because of the nonnegative bounds utilized. This is one of the advantages of the variational QPE approach.

When the a priori statistics provide no information, the performance of the variational QPE approach is comparable with G13's method, generally. Nevertheless, the advantage of the variational QPE approach is its flexibility. Additional information can be incorporated through the a priori or the upper and lower 
constraints easily. The impact of the a priori on the $R$ estimation will be shown in section $2 \mathrm{~d}$.

\section{d. Impact of the a priori}

As shown in Fig. 7a, the a priori estimated from the simulated $Z_{\mathrm{H}}$ in Fig. 4 shows some deviation from the intrinsic $R$ because of both the DSD variability and the random noise. The corresponding error bars denote the uncertainty $\sigma_{R \text {,ap }}$. With incorporating the information of $Z_{\mathrm{H}}$ into the variational analysis as the a priori, the statistical performance of the estimated $R$ is shown in Figs. $7 \mathrm{~b}$ and $7 \mathrm{c}$. Compared to the results where the a priori provides no information (see Fig. 6), the mean CC increases and both the mean RMSE and NE decrease. Another significant improvement is that the uncertainty $\left(\sigma_{R}\right)$ of the $R$ estimation decreases (gray shading in Fig. 7b; red line in Fig. 7c). Although there is significant uncertainty in $R_{Z_{\mathrm{H}}}$, we can obtain more confident $R$ estimates with the combination of the a priori and the measurements $\left(\Phi_{\mathrm{DP}}\right)$. The accuracy of the $R$ estimation is obviously better and its uncertainty $\left(\sigma_{R}\right)$ is less than the estimation based on G13's method. There is also a shortcoming in the $R$ estimates. Because of the sensitivity of the $Z-R$ relation to the DSD properties, the $R\left(Z_{\mathrm{H}}\right)$ tends to underestimate the rainfall rate. This effect is also included in the variational analysis. However, the underestimation of the results of the variational analysis is not significant and is acceptable when we take the decease of the uncertainty $\left(\sigma_{R}\right)$ of the $R$ estimation into consideration. We will investigate how the inclusion of the a priori affects the QPE performance in real cases in section 4.

\section{Verification with the data of an S-band polarimetric radar}

\section{a. Data and the quality control}

In Guangdong Province, an S-band polarimetric radar (called Guangzhou S-POL) is deployed at the city of Guangzhou. This radar is part of the Chinese operational radar network, whose main configuration is shown in Table 1. Guangzhou S-POL was a Doppler weather radar built several years ago, and it has been upgraded with polarimetric capability since 2016. In May and June 2016, about seven precipitation events occur within the coverage of Guangzhou S-POL. In this section QPE based on the aforementioned variational approach is verified using these events.

Guangzhou, one of China's most developed cities, is still undergoing rapid development, so the topographic condition around the radar site changes every year. The radar observations suffer from ground clutter
TABLE 1. Settings and parameters of Guangzhou S-POL and its observations.

\begin{tabular}{ll}
\hline \multicolumn{1}{c}{ Parameters } & \multicolumn{1}{c}{ Guangzhou S-POL } \\
\hline Transmitter & $2.885 \mathrm{GHz}$ (klystron) \\
PRF & $322-1282$ \\
Pulse width & $1.57 \mu \mathrm{s}$ \\
Peak power & $700 \mathrm{~kW}$ \\
Receiver & Simultaneous horizontal/vertical \\
Noise figure & $<3 \mathrm{~dB}$ \\
Dynamic range & $95 \mathrm{~dB}$ \\
Antenna feeder & Paraboloid, center feed \\
Antenna gain & $44.7 \mathrm{~dB}$ \\
Antenna aperture & $8.5 \mathrm{~m}$ \\
Beamwidth & $0.95^{\circ}$ \\
Polarimetric mode & Simultaneous horizontal and vertical \\
& transmit and receive \\
Scanning mode & PPI: $0^{\circ}-360^{\circ}$, rotating speed: $11^{\circ}-16^{\circ} \mathrm{s}^{-1}$, \\
& time for volume coverage patterns \\
& $(\mathrm{VCP}): \sim 6$ min \\
Elevations & $0.5,1.5,2.4,3.3,4.3,6.0,9.9,14.6,19.5$ \\
Precision & \\
Range resolution & $0.25 \mathrm{~km}$ \\
Radar variables & $Z_{\mathrm{H},}, Z_{\mathrm{DR}}, \Phi_{\mathrm{DP}}, \rho_{\mathrm{hv}}$, SNR, $v_{r}, \sigma_{v}$ \\
$Z_{\mathrm{H}}$ precision & $1.0 \mathrm{~dB}$ \\
$Z_{\mathrm{DR}}$ precision & $0.2 \mathrm{~dB}$ \\
$\Phi_{\mathrm{DP}}$ precision & $2.0^{\circ}$ \\
$v_{r}$ precision & $1.0 \mathrm{~m} \mathrm{~s}{ }^{-1}$ \\
\hline &
\end{tabular}

contamination resulting from the tall buildings, the TV towers, and so on. Ground clutter contamination needs to be taken into consideration in QPE. Algorithms such as ground clutter detection and mitigation techniques are usually employed in radar digital signal processing (DSP) to mitigate the impact of ground clutter (Doviak and Zrnić 1993; Zhang 2016). Nevertheless, its impact on the radar measurements can seldom be fully eliminated, especially when the power of the ground clutter is dominant or the radial velocity of precipitation is close to zero. For polarimetric radars, the ground clutter mitigation can make the signals of the horizontal and the vertical channels incoherent/ unbalanced. Thus, the accuracy of $Z_{\mathrm{DR}}, \Phi_{\mathrm{DP}}$, and $\rho_{\mathrm{hv}}$ measurements, which are defined as the power ratio, the phase difference, and the correlation of the horizontal and the vertical signals, respectively, will probably decrease when the echo is contaminated by ground clutter. As we know, $\Phi_{\mathrm{DP}}$ is based on the propagation effect of electromagnetic wave. Besides, $Z_{\mathrm{DR}}$ is significantly affected by ground clutter, especially when the signal of the weather echoes is weaker than the clutter. QPE with the variational approach using $\Phi_{\mathrm{DP}}$ and $Z_{\mathrm{H}}$ proposed in this paper is considered to be less affected by the clutter than QPE with the algorithms with $Z_{\mathrm{DR}}$ used. 

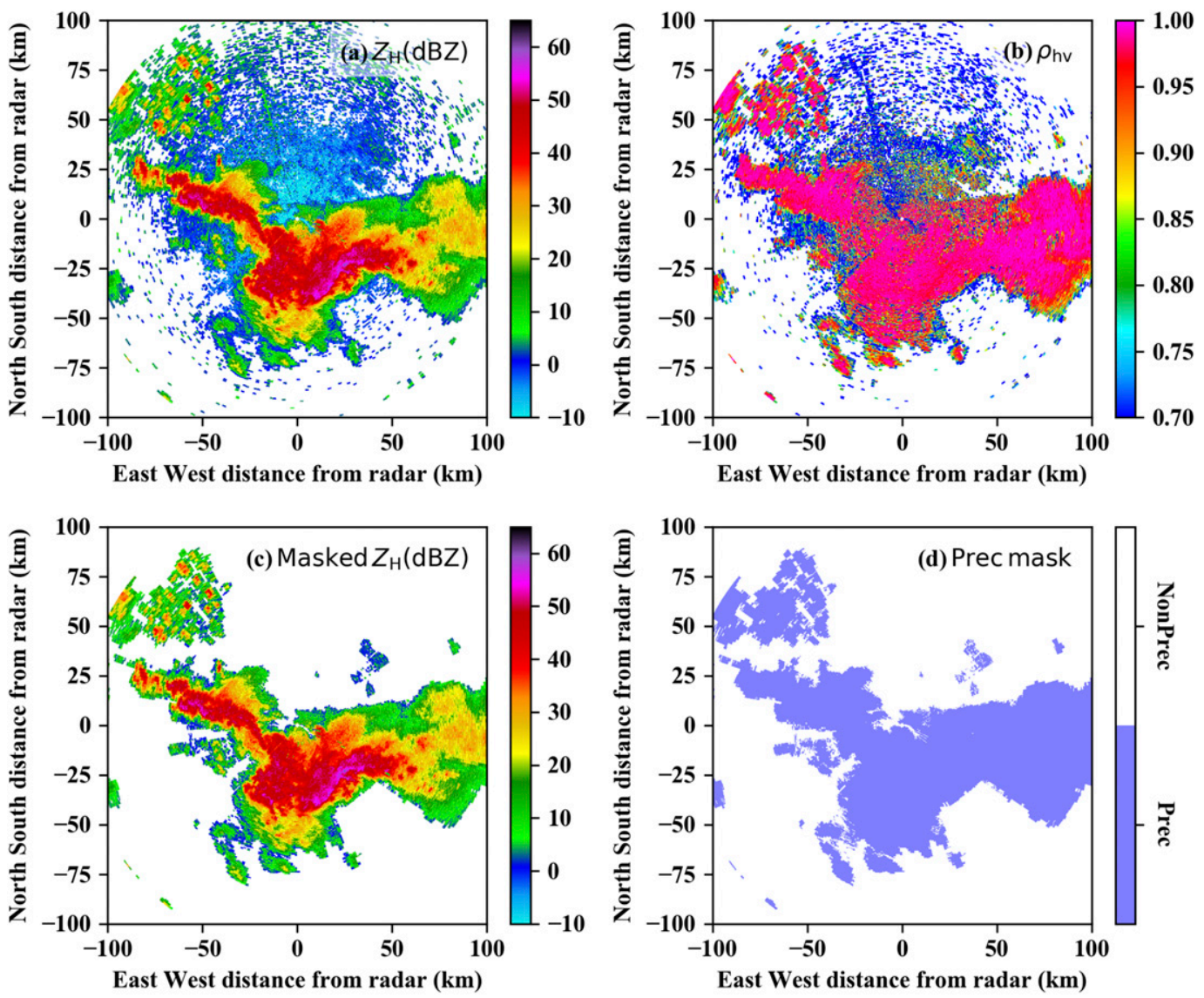

FIG. 8. The PPI images of a precipitation event collected by Guangzhou S-POL at 0030 UTC 10 May 2016 at the elevation angle of $1.5^{\circ}$. (a) $Z_{\mathrm{H}}(\mathrm{dBZ})$, (b) $\rho_{\mathrm{hv}}$, (c) $Z_{\mathrm{H}}$ after the removal of nonprecipitation echoes, and (d) the mask utilized to separate the precipitation and nonprecipitation echoes.

Before being used in QPE, the quality of the data from Guangzhou S-POL is carefully controlled via the following four steps. As documented by Chen et al. (2014), the internal calibration system of Guangzhou S-POL using internally generated test signals and routine maintenance guarantee the $Z_{\mathrm{H}}$ precision to be within $1 \mathrm{~dB}$.

1) Echoes with $\rho_{\mathrm{hv}}$ less than 0.85 are identified as potential nonmeteorological echoes. If the areas of the nonmeteorological echoes embedded inside the meteorological echoes are small with the number of clustered gates less than 20, they are marked back to precipitation. Some nonmeteorological echoes can have large $\rho_{\text {hv }}$ greater than 0.85 as a result of the random measurement errors. Similarly, if the echo area has fewer than 20 clustered gates, they are removed utilizing a de-speckle filter.

2) Term $Z_{\mathrm{DR}}$ is calibrated using light rain observations. The averaged intrinsic $Z_{\mathrm{DR}}$ value of the light drizzle echo with $Z_{\mathrm{H}}$ between $10 \sim 20 \mathrm{dBZ}$ is assumed to be very close to $0 \mathrm{~dB}$ (Giangrande and Ryzhkov 2005).
The difference of the averaged value of the observed $Z_{\mathrm{DR}}$ and the intrinsic value of the light rain region is considered as the $Z_{\mathrm{DR}}$ bias.

3) The system $\Phi_{D P}$ is estimated from the statistics of the $\Phi_{\mathrm{DP}}$ values of the meteorological echo close to the radar site.

4) For meteorological echoes, if the difference of the $\Phi_{\mathrm{DP}}$ values at two neighboring range gates is greater than $35^{\circ}$, then the $\Phi_{\mathrm{DP}}$ measurements at these two gates are considered to be contaminated by clutter and removed. After the unfolding, the data at these gates are refilled by linearly interpolating the $\Phi_{\mathrm{DP}}$ measurements at the surrounding gates.

A plane position indication (PPI) image of radar data on 10 May 2016 at the elevation angle of $1.5^{\circ}$ is shown in Fig. 8. The echoes to the north of the radar site characterized with low $\rho_{\mathrm{hv}}$ are probably from nonprecipitation scatterers. In our processing, only the precipitation echoes are retained (see Fig. 8d). The precipitation echoes contaminated by ground 

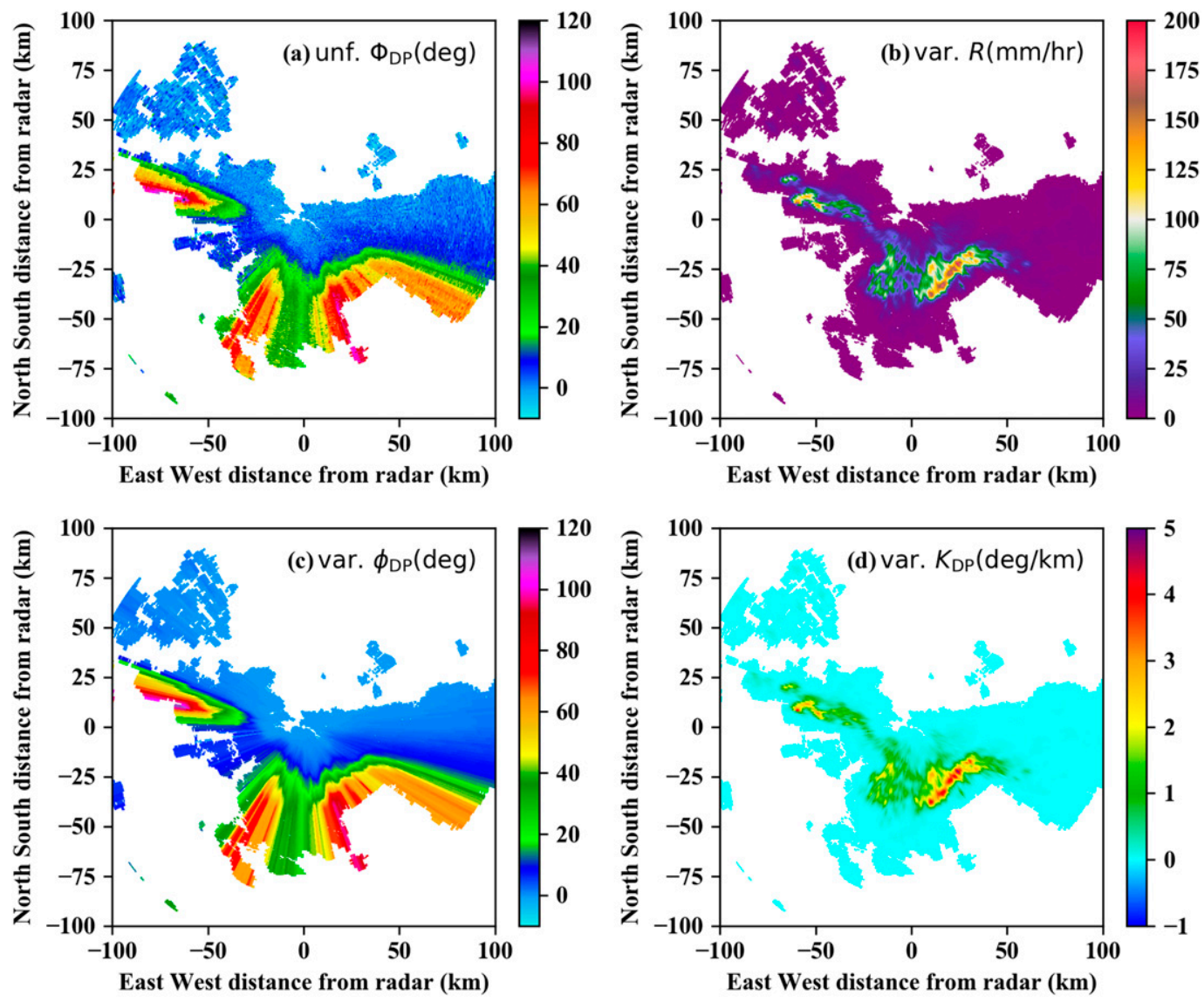

FIG. 9. The retrieval results for the radar scan shown in Fig. 8. (a) The unfolded $\Phi_{\mathrm{DP}}$ after the removal of the system phase, and (b) the optimized $R$ from the proposed variational approach. (c) The optimized $\phi_{\mathrm{DP}}$ and (d) the optimized $K_{\mathrm{DP}}$ are shown.

clutter near the radar site are also processed with the clutter mitigation algorithms in the DSP. It causes inconsistency in the signals from the horizontal and vertical channels. The corresponding $\rho_{\mathrm{hv}}$ values decrease and the reliability of the radar measurements also decreases. Besides, the large backscattering phase from hail will also bring in uncertainty. Thus, the removal and the refill of the $\Phi_{\mathrm{DP}}$ measurements contaminated by ground clutter or hail (see step 4) will ameliorate the rain measurements.

After quality control, the errors of $Z_{\mathrm{H}}, Z_{\mathrm{DR}}$, and $\Phi_{\mathrm{DP}}$ are assumed to be equal to $1 \mathrm{~dB}, 0.2 \mathrm{~dB}$, and $2.0^{\circ}$, respectively, according to the configuration of the radar (Table 1). The rainfall rates for the radar scans are optimized based on the variational approach with $R\left(Z_{\mathrm{H}}\right)$ as the a priori. The results for the same radar scan shown in Fig. 8 are shown in Fig. 9. The main precipitation core (southeast of the radar site) is characterized with large $Z_{\mathrm{H}}$ and a sharp radial increase in $\Phi_{\mathrm{DP}}$. The optimized rainfall rates (Fig. 9b) of this region are over $150 \mathrm{~mm} \mathrm{~h}^{-1}$. The intermediate variables $\phi_{\mathrm{DP}}$ and $K_{\mathrm{DP}}$ are also shown.
The optimized $\phi_{\text {DP }}$ values (Fig. 9c) are consistent with the unfolded $\Phi_{\mathrm{DP}}$ measurements (Fig. 9a). Most of the random errors are removed and the $\phi_{\mathrm{DP}}$ values are also consistent in the azimuthal direction. The optimized $K_{\text {DP }}$ values (Fig. 9d) of the main precipitation core are over $3^{\circ} \mathrm{km}^{-1}$. There is no negative value in $K_{\mathrm{DP}}$, which indicates that the optimization in our study follows the physical constraints. This is an evident advantage of this method as compared with the conventional least squares fit based $K_{\mathrm{DP}}$ estimation and QPE (Huang et al. 2017).

Besides the proposed variational approach with $R\left(Z_{\mathrm{H}}\right)$ included as the a priori (notated with VWAP), the rainfall rates for the radar scans are also estimated with three other approaches. To show the impact of the a priori, the results for the variational approach with no additional information used in a priori (notated with VWOAP) are utilized as references. The variational approach proposed by $\mathrm{H} 07$ (notated with $\mathrm{VH} 07$ ) is also employed to show the impact of different forward operators. In $\mathrm{VH} 07$, the forward operator is based on the gamma distribution with a fixed $\mu=3$, and the statistical 
terminal velocity relation is the same as that used by Brandes et al. (2002). And last, the power-law estimator $R=57.132\left|K_{\mathrm{DP}}\right|^{0.785} \operatorname{sign}\left(K_{\mathrm{DP}}\right)$ based on the least squares fitted $K_{\mathrm{DP}}$ (notated with RKDP) is also included. The configuration of the least squares fitting is the same as that used in Huang et al. (2017) except that the fitting window for a gate with $Z_{\mathrm{H}}$ below $40 \mathrm{dBZ} Z$ is set to $9 \mathrm{~km}$. The notation $\operatorname{sign}(\cdot)$ means the sign of $K_{\mathrm{DP}}$ is included in the rainfall-rate calculation as proposed by Ryzhkov and Zrnić (1996). In later subsections, the QPEs based on these four different approaches are compared against the rain gauge observations to show their performance. The radar-estimated rainfall rates above the gauge sites are extracted and temporally integrated into the accumulated rainfall amounts (ARs) of different temporal resolutions, which facilitate the quantitative comparisons. To mitigate the impact of the ground clutters and the partial beam blockage on the radar QPEs, the scans at the $1.5^{\circ}$ elevation are utilized. On the other hand, there are more than 600 rain gauges within a distance of $75 \mathrm{~km}$ from the radar site. To make a convincing and objective comparison, the quality of the gauge data is controlled in advance using a method of speckle filtering (Lee et al. 1999). The spatial continuity of the accumulated rainfall amounts is checked and the outliers are probably from the unreliable gauge sites. These sites are excluded from the QPE evaluation.

\section{b. QPEs in one case}

First, a precipitation event on 9-10 May 2016 is selected for the QPE evaluation. The event caused the maximum accumulated rainfall, over $150 \mathrm{~mm}$, in southern China. The rain gauges' derived total ARs of the whole event $\left(\mathrm{AR}_{\text {gauge }}\right)$ are shown in Fig. 10. The relative differences between the ARs based on VWAP (AR $\left(\mathrm{R}_{\mathrm{VWAP}}\right)$ and $\mathrm{AR}_{\text {gauge }}$, defined as $\left(\mathrm{AR}_{\mathrm{VWAP}}-\mathrm{AR}_{\text {gauge }}\right) / \mathrm{AR}_{\text {gauge }}$, are also included. The heavy rainfall band extended from northwest to southeast with the core located near the radar site. There is a slight underestimation in the radarderived total ARs near the radar site (within the range of $20 \mathrm{~km}$ ) and a slight overestimation outside the range of $30 \mathrm{~km}$ from the radar. The biases of the radar-derived ARs and the rain gauge-based ARs are caused by the mismatch of the sampling volumes, the vertical microphysical structure change or horizontal propagation when rain falls from the radar sampling volumes to the ground, the interstorm DSD variability, the quality of the radar data and the rain gauge data, the uncertainty in the QPE algorithms, and so on. Overall, the underestimation or overestimation of the total ARs is relatively slight.

The radar hourly rainfall estimates for all the gauge sites denoted in Fig. 10 on the basis of the four approaches are intercompared, as shown in Fig. 11. The performances of the different approaches vary.

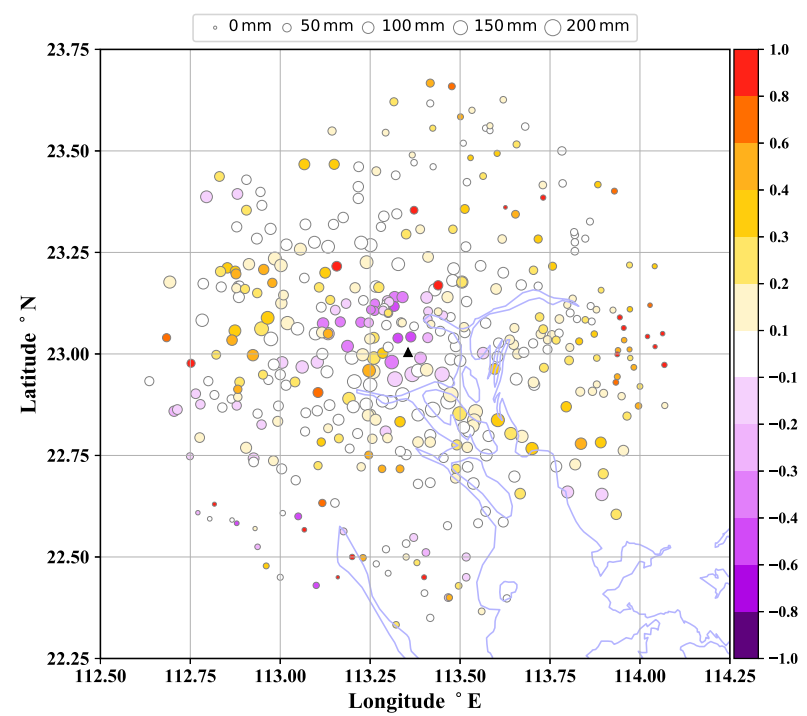

FIG. 10. The difference in the total accumulated rainfall estimated from the rain gauges and the radar-derived total accumulated rainfall (the elevation angle of $1.5^{\circ}$ ) based on the proposed variational approach from the precipitation event from 0000 UTC 9 May to 1200 UTC 10 May 2016. The places where the rain gauges were deployed (circles). The size of the circles denotes the total accumulated rainfall from the rain gauges, and the relative difference (shading). The radar site position (black triangle) is marked.

Generally, VWAP has the best performance among the four approaches. It shows a high correlation coefficient $(\mathrm{CC}=0.94)$ and relative low errors $(\mathrm{RMSE}=3.38 \mathrm{~mm}$, $\mathrm{NE}=0.34)$. When no information is provided as the a priori, VWOAP still shows satisfactory performance. The correlation coefficient decreases slightly $(\mathrm{CC}=0.92)$ and the errors slightly increase (RMSE $=3.66 \mathrm{~mm}$, $\mathrm{NE}=0.44)$, which demonstrates the positive effect of the inclusion of $R\left(Z_{\mathrm{H}}\right)$ as the a priori. For $\mathrm{VH} 07$, all three kinds of radar measurements $\left(Z_{\mathrm{H}}, Z_{\mathrm{DR}}\right.$, and $\left.\Phi_{\mathrm{DP}}\right)$ are utilized, while the performance of the rainfall estimation is roughly comparable with VWOAP, in which only $\Phi_{\mathrm{DP}}$ measurements are used for QPE. For the conventional RKDP method, even after the temporal accumulation, negative rainfall estimates may exist, which may introduce extra uncertainty (Huang et al. 2017). As a consequence, RKDP has the worst performance in the hourly rainfall estimation.

Figure 12 shows the comparisons of the ARs derived from the four approaches and the rain gauge observations at each gauge site. Generally, the site-by-site comparisons show that the hourly ARs estimated with VWAP are in good agreement with those observed by the rain gauges, with the averaged $\mathrm{CC}$ being 0.95 and the averaged NE being 0.45 . VH07 is also satisfactory in this situation, but it is not as good as VWAP. The results for RKDP not surprisingly show the worst performance. Especially at 

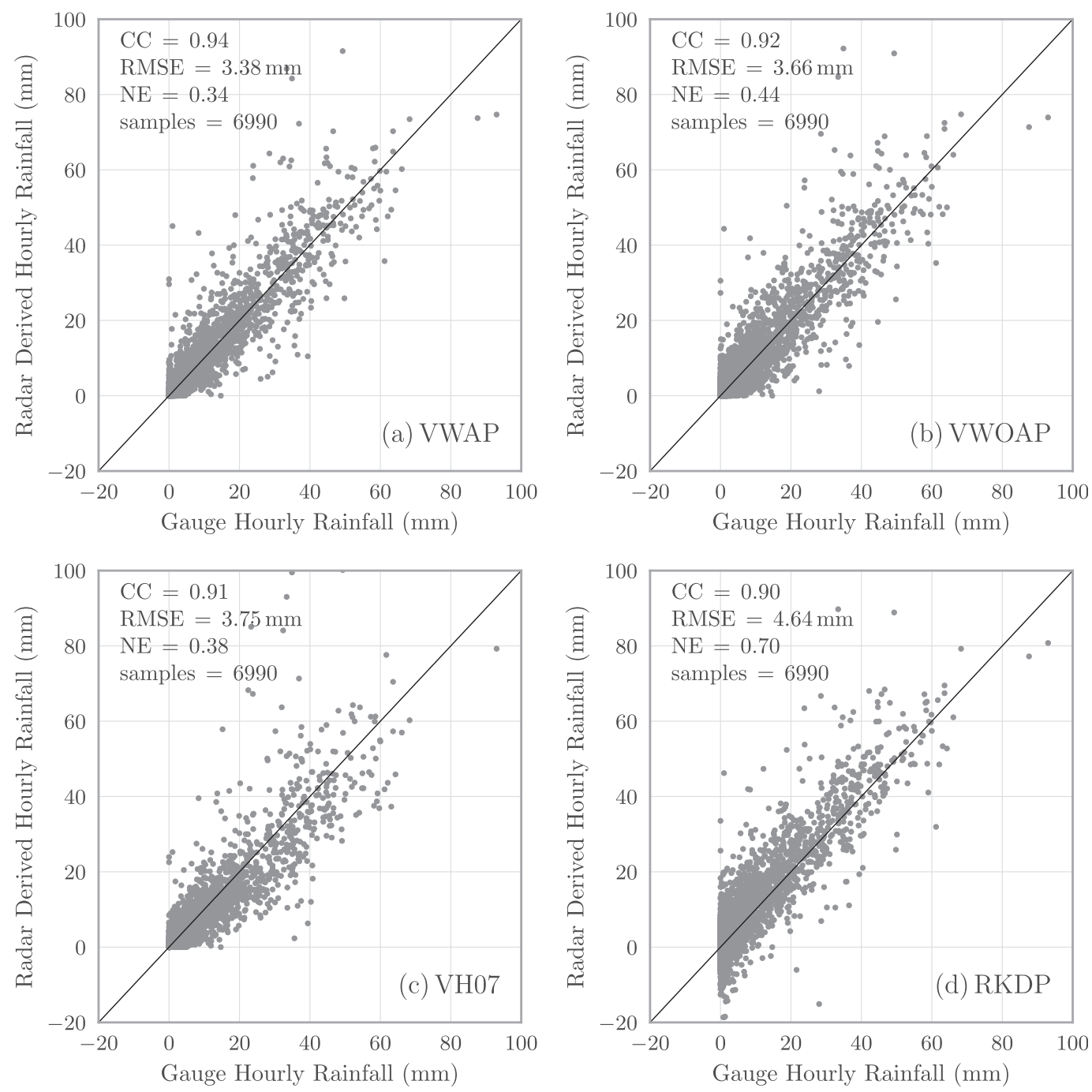

FIG. 11. Scatterplot of the hourly rainfalls observed by all the rain gauges (shown in Fig. 10) vs the hourly rainfall estimates from the PPI scans at the elevation angle of $1.5^{\circ}$ based on (a) VWAP, (b) VWOAP, (c) VH07, and (d) RKDP.

the gauge sites with lighter ARs (see Fig. 10), the results for RKDP have low CCs and high NEs.

\section{c. QPEs in all seven events}

To illustrate the case dependence of different QPE approaches, the CCs, RMSEs, and NEs for the hourly rainfall comparisons for all seven rainfall events are shown in Table 2. The QPE accuracies vary in different rainfall events, probably because of the DSD variability and different durations. However, VWAP generally has the best performance in all the events and RKDP has the worst performance. VWOAP and VH07 have comparable performance according to Table 2 . The radar QPE in the event at $0400 \sim 0900$ UTC 7 June exhibits the worst accuracy compared with the other events, mainly because of the short duration (about $5 \mathrm{~h}$ ) and the smaller sampling number (about 500).

Figure 13 shows the comparison between the radarderived hourly rainfall amounts from all the cases compared and those observed by gauges. Similar conclusions can be drawn from the comparison. The total sampling number is more than 40000 , which guarantees the objectivity of the evaluation. For VWAP and VWOAP, the hourly rainfall amounts are generally symmetrically distributed along the identity line. But for VH07 and RKDP, the results are not as good as the results for VWAP and VWOAP. The hourly rainfall amounts estimated based on VWAP coincide best with the gauge-based results, with CC being 0.92, RMSE being $2.52 \mathrm{~mm}$, and NE being 0.42 . For VWOAP, the 


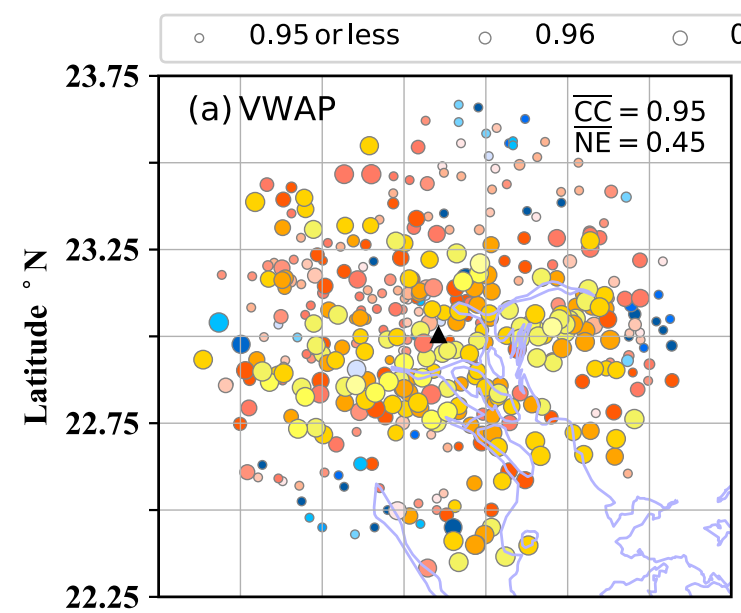

$\begin{array}{lllllll}0.97 & \circ & 0.98 & 0 & 0.99 & \bigcirc & 1 .\end{array}$
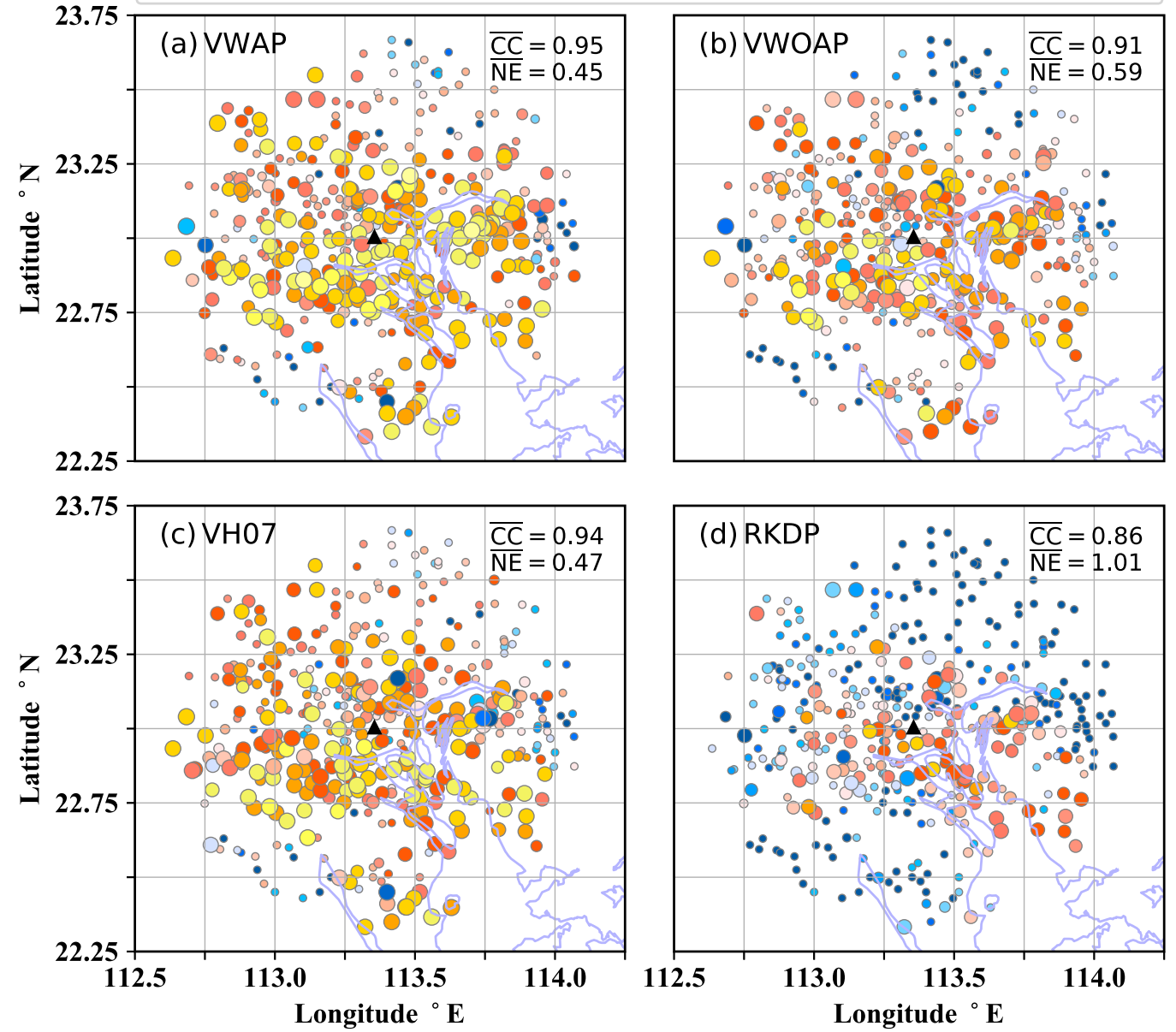

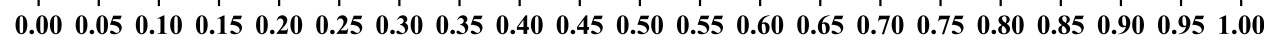

FIG. 12. Hourly rainfall comparisons at the rain gauge sites for (a) VWAP, (b) VWOAP, (c) VH07, and (d) RKDP. The places where the rain gauges were deployed (circles). The size of the circles represents the correlation coefficient between the time series of the radar-derived ARs and the time series of the gauge-derived ARs, and the the normalized error between them (shading).

results get a little bit worse than the results for VWAP. The difference mainly exists when the gauge-derived hourly rainfall amounts are less than $10 \mathrm{~mm}$. The results based on VH07 are comparable with VWOAP, with similar values of CC, RMSE, and NE. The conventional power-law estimator-based QPE (RKDP) exhibits the worst performance. The CC is only 0.83 , RMSE is about $4.1 \mathrm{~mm}$, and NE is very large (1.02). The negative hourly rainfall amounts for RKDP mainly occur when the precipitation is light. From the gauge observation, most of the hourly rainfall amounts are less than $10 \mathrm{~mm}$. Thus, negative $K_{\mathrm{DP}}$ values estimated from the least squares fitting method have a very large impact on QPE.

The QPE evaluations are also separated into two groups as shown in Table 3. The evaluation for the gauge-derived hourly rainfall amounts less than $10 \mathrm{~mm}$ represents the result for light rain. VWAP still shows the best performance with the highest $\mathrm{CC}$ and the lowest RMSE/NE. Meanwhile, the performance of VWOAP worsens as a result of the lack of information from $Z_{\mathrm{H}}$ measurements. For light rain, the useful information in $\Phi_{\mathrm{DP}}$ is mainly outweighed by measurement errors. Nevertheless, the variational analysis still results in better QPE than the conventional RKDP, since the least squares fitting method has no capability to constrain $K_{\mathrm{DP}}$ within the physical limits. For VH07, the performance is between VWAP and VWOAP. The evaluation for the gauge-derived hourly rainfall amounts greater than $10 \mathrm{~mm}$ is for heavy rain. VWOAP and RKDP now have similar performance, mainly because the least 
TABLE 2. Case-by-case comparisons of the hourly rainfall amounts estimated based on four approaches and those derived from the rain gauges.

\begin{tabular}{|c|c|c|c|c|c|c|c|c|}
\hline & VWAP & VWOAP & VH07 & RKDP & VWAP & VWOAP & VH07 & RKDP \\
\hline Time & \multicolumn{4}{|c|}{0000 UTC 9 May $\sim 1200$ UTC 10 May } & \multicolumn{4}{|c|}{19 May 1700 UTC $\sim 0300$ UTC 21 May } \\
\hline $\mathrm{CC}$ & 0.94 & 0.92 & 0.91 & 0.90 & 0.91 & 0.87 & 0.88 & 0.75 \\
\hline RMSE & 3.38 & 3.66 & 3.75 & 4.64 & 2.08 & 2.5 & 2.53 & 4.08 \\
\hline $\mathrm{NE}$ & 0.34 & 0.44 & 0.38 & 0.70 & 0.45 & 0.61 & 0.56 & 1.27 \\
\hline Time & \multicolumn{4}{|c|}{0000 UTC 27 May 1200 UTC 28 May } & \multicolumn{4}{|c|}{0300 UTC 4 Jun $\sim 1100$ UTC 4 Jun } \\
\hline $\mathrm{CC}$ & 0.94 & 0.89 & 0.9 & 0.77 & 0.91 & 0.90 & 0.87 & 0.84 \\
\hline RMSE & 1.60 & 2.09 & 2.15 & 3.41 & 3.80 & 4.01 & 4.22 & 5.60 \\
\hline $\mathrm{NE}$ & 0.43 & 0.64 & 0.64 & 1.52 & 0.55 & 0.54 & 0.64 & 0.95 \\
\hline Time & \multicolumn{4}{|c|}{1800 UTC 4 Jun $\sim 1300$ UTC 5 Jun } & \multicolumn{4}{|c|}{0400 UTC 7 Jun $\sim 0900$ UTC 7 Jun } \\
\hline $\mathrm{CC}$ & 0.92 & 0.88 & 0.83 & 0.82 & 0.86 & 0.81 & 0.84 & 0.79 \\
\hline RMSE & 2.12 & 2.42 & 2.87 & 3.58 & 3.86 & 4.57 & 3.45 & 5.54 \\
\hline $\mathrm{NE}$ & 0.41 & 0.47 & 0.53 & 0.80 & 0.65 & 0.91 & 0.58 & 1.36 \\
\hline Time & \multicolumn{4}{|c|}{2000 UTC 27 Jun $\sim 1100$ UTC 28 Jun } & & & & \\
\hline $\mathrm{CC}$ & 0.88 & 0.84 & 0.87 & 0.80 & & & & \\
\hline RMSE & 3.16 & 3.64 & 3.23 & 4.61 & & & & \\
\hline $\mathrm{NE}$ & 0.38 & 0.53 & 0.48 & 0.86 & & & & \\
\hline
\end{tabular}

squares fitted $K_{\mathrm{DP}}$ gets more accurate as the rainfall gets heavier. The results based on VWAP are still better than VWOAP, which means the $Z_{\mathrm{H}}$ measurements still positively contribute to QPE. VH07 now has the worst performance, mainly because it is not tuned for southern China and its forward operator cannot capture the DSD characteristics of these rainfall events well.

\section{Conclusions and discussion}

In this study a variational QPE approach based on $\Phi_{\mathrm{DP}}$ measurements is proposed. With the physical constraints utilized in the variational approach, erroneous negative rainfall-rate and $K_{\mathrm{DP}}$ estimates (which is an evident disadvantage for the conventional least squares fitting-based $K_{\mathrm{DP}}$ estimation and QPE) no longer exist. The introduction of the range smoothing filter is essentially a utilization of the continuity of the precipitation system and the spatial coherence of the rainfall rate, which makes the rainfall-rate estimates suffer less from the random errors in $\Phi_{\mathrm{DP}}$ measurements. However, oversmoothing will oversuppress the physical variation in the rainfall rates. The best smoothing factor is determined through the comparison experiments. The QPE is further improved via incorporating the rainfall rates estimated from $Z_{\mathrm{H}}$ into the variational analysis as the a priori information. The weighting of the information from the $\Phi_{\mathrm{DP}}$ measurements and the a priori is decided by their statistical errors. This has been demonstrated via an idealized case in section 3 . With the a priori utilized, the results from the variational approach outperform those from the linear programming method.
Four QPE approaches are applied to the observations of Guangzhou S-POL in May and June 2016 to show the performance of our proposed variational approach. Generally, the VWAP results are the best rainfall estimates because of the integrated utilization of $Z_{\mathrm{H}}$ and $\Phi_{\mathrm{DP}}$ measurements. VH07 is on the basis of the $Z-R$ relation and the gamma distribution with a fixed $\mu$. It has better performance than VWOAP when the rainfall is light [RA(gauge) $<10 \mathrm{~mm}$ ] and more information is available from the measurements $\left(Z_{\mathrm{H}}, Z_{\mathrm{DR}}\right.$, and $\left.\Phi_{\mathrm{DP}}\right)$. However, even when only $\Phi_{\mathrm{DP}}$ measurements are used in VWOAP, QPE results are still better than VH07 when the rainfall is heavier [RA(gauge) $>10 \mathrm{~mm}$ ], probably because of the inaccurate forward operator of VH07. The QPE results based on the conventional RKDP do not coincide well with the gauge-based results, especially when the rainfall is light. Unphysical negative rainfall amounts can result from the negative least squares-fitted $K_{\mathrm{DP}}$ values. For heavier rainfall, RKDP shows comparable performance to VWOAP, but it is still not as good as VWAP.

According to the case-by-case evaluation (Table 2), QPE performance based on VWAP and VWOAP varies mainly because of the uncertainties in the statistical $R-K_{\mathrm{DP}}$ relation and the $Z_{\mathrm{H}}-R$ relation. Different weather systems usually have different DSD characteristics and cannot be accurately characterized with fixed relations. Of course, if the microphysics of the weather system is very different from the climatic statistics and the statistical $R-K_{\mathrm{DP}}$ and $Z_{\mathrm{H}}-R$ relations are not appropriate for characterization of the system, then the performance of the variational approach will be very poor. Nevertheless, the advantage of the forward operator in 

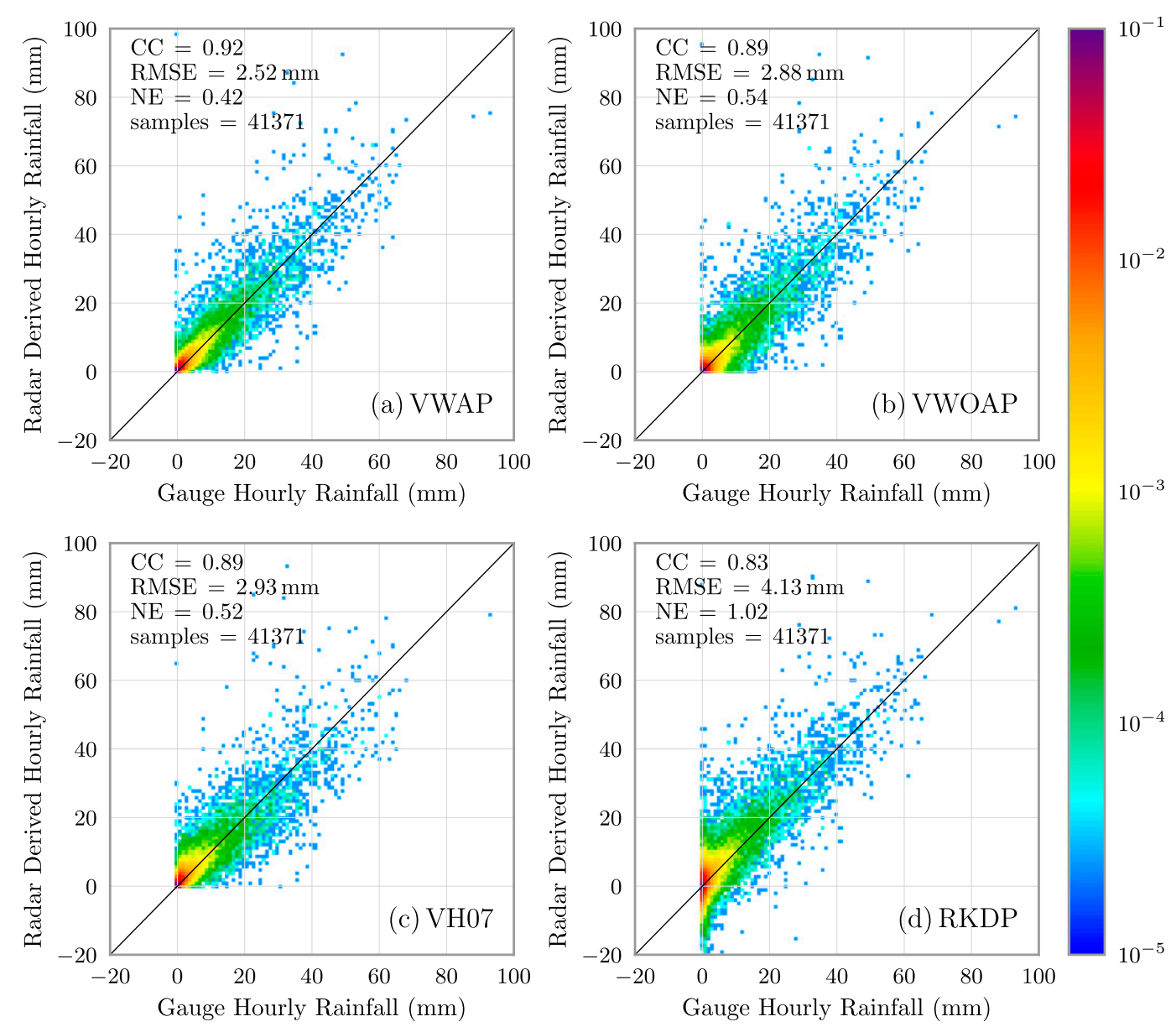

FIG. 13. Integrated comparisons of the hourly rainfalls derived from the gauges vs the hourly rainfall estimates from the PPI scans at the elevation angle of $1.5^{\circ}$ based on (a) VWAP, (b) VWOAP, (c) VH07, and (d) RKDP for all seven cases listed in Table 2. The probability densities of the samples (shading), for which the category sizes of the rainfall are $1 \mathrm{~mm}$.

the proposed approach is that $K_{\mathrm{DP}}$ is approximately the 4.2nd moment of DSD, and the $R-K_{\mathrm{DP}}$ relation should not change very significantly from the climatic statistics. The inclusion of $Z_{\mathrm{DR}}$ may help to reduce the impact of DSD uncertainty in the calculation of the a priori. However, the measurement errors of $Z_{\mathrm{DR}}$ may deteriorate the accuracy of the a priori. The $0.2-\mathrm{dB}$ error in $Z_{\mathrm{DR}}$ can result in about $20 \%$ error in rainfall-rate estimates (Lee 2006). The accuracy of $Z_{\mathrm{DR}}$ measurement is affected by the radar hardware, the surrounding environment, attenuation, and many other factors. Yet, the calibration of $Z_{\mathrm{DR}}$ is not an easy task. It is not easy to ensure the accuracy of $Z_{\mathrm{DR}}$ and to characterize the error of the a priori if $Z_{\mathrm{DR}}$ is included. We use only $R\left(Z_{\mathrm{H}}\right)$ as the a priori, and the uncertainties resulting from DSD variability and measurement errors are also considered as in the a priori error covariance matrix. Comparative studies in section 4 have demonstrated that the combination of the forward operator and the a priori helps to improve radar QPE. The variational approach should

TABLE 3. Comparisons of the hourly rainfall amounts based on four approaches and those derived from the rain gauges for all seven cases listed in Table 2 under two different conditions, i.e., $\mathrm{RA}$ (gauge) $\leq 10 \mathrm{~mm}$ and $\mathrm{RA}$ (gauge) $>10 \mathrm{~mm}$.

\begin{tabular}{lcccccrrr}
\hline \hline & VWAP & VWOAP & VH07 & RKDP & VWAP & VWOAP & VH07 & RKDP \\
\hline Condition & \multicolumn{9}{c}{ RA(gauge) $\leq 10 \mathrm{~mm}$} & & \multicolumn{3}{c}{ RA(gauge) $>10 \mathrm{~mm}$} \\
CC & 0.77 & 0.64 & 0.74 & 0.51 & 0.81 & 0.79 & 0.75 & 0.79 \\
RMSE & 1.67 & 2.10 & 2.02 & 3.70 & 7.18 & 7.62 & 8.12 \\
NE & 0.66 & 0.91 & 0.86 & 2.07 & 0.25 & 0.27 & 0.28 & 0.27 \\
\hline
\end{tabular}


result in satisfactory QPE unless the DSD properties of the events are extremely different from the climatic statistics, which will also be a problem in other QPE approaches.

As we know, all kinds of radar QPE approaches suffer from two sources of uncertainties and errors, that is, the uncertainties of estimation models and the measurement errors. Usually, the combination of polarimetric variables can result in better estimation models, while QPE may be affected more by the measurement errors. Composing different rainfall estimators according to the rain type classification (e.g., separation of stratiform and convective precipitation or identification of coalescencedominant precipitation) and the error analysis is a promising way to minimize the impact of uncertainties and errors (Porcacchia et al. 2017; Steiner et al. 1995). It will be included in the current variational QPE framework and discussed in future work.

Acknowledgments. This work was primarily supported by the National Key Research and Development Program of China under Grant 2017YFC1501703, the National Fundamental Research 973 Program of China (2013CB430101), the National Natural Science Foundation of China (Grants 41475015 and 41322032), the Open Research Program of the State Key Laboratory of Severe Weather, and the Beijige Open Research Fund for Nanjing Joint Center of Atmospheric Research. The authors thank Dr. Bowen Zhou (NJU) for the careful reading and editing of the manuscript. The authors also thank the scientists and engineers of NJU-2DVD, Guangzhou S-POL, and other instruments. The data are archived on a specialized computer at Nanjing University and are managed by the project office (yang.zhengwei@nju.edu.cn). These data can be accessed upon request, but their usage may be restricted by relevant Chinese government rules and regulations that are beyond the control of the authors.

\section{REFERENCES}

Berne, A., and W. F. Krajewski, 2013: Radar for hydrology: Unfulfilled promise or unrecognized potential? Adv. Water Resour., 51, 357-366, https://doi.org/10.1016/j.advwatres. 2012.05.005.

Brandes, E. A., G. Zhang, and J. Vivekanandan, 2002: Experiments in rainfall estimation with a polarimetric radar in a subtropical environment. J. Appl. Meteor., 41, 674-685, https:// doi.org/10.1175/1520-0450(2002)041<0674:EIREWA>2.0.CO;2.

Bringi, V. N., and V. Chandrasekar, 2001: Polarimetric Doppler Weather Radar: Principles and Applications. Cambridge University Press, $664 \mathrm{pp}$.

, C. R. Williams, M. Thurai, and P. T. May, 2009: Using dualpolarized radar and dual-frequency profiler for DSD characterization: A case study from Darwin, Australia. J. Atmos. Oceanic Technol., 26, 2107-2122, https://doi.org/10.1175/2009JTECHA1258.1.
—, M. A. Rico-Ramirez, and M. Thurai, 2011: Rainfall estimation with an operational polarimetric C-band radar in the United Kingdom: Comparison with a gauge network and error analysis. J. Hydrometeor., 12, 935-954, https://doi.org/10.1175/ JHM-D-10-05013.1.

Byrd, R. H., P. Lu, J. Nocedal, and C. Zhu, 1995: A limited memory algorithm for bound constrained optimization. SIAM J. Sci. Comput., 16, 1190-1208, https://doi.org/10.1137/0916069.

Cao, Q., G. Zhang, E. A. Brandes, and T. J. Schuur, 2010: Polarimetric radar rain estimation through retrieval of drop size distribution using a Bayesian approach. J. Appl. Meteor. Climatol., 49, 973-990, https://doi.org/10.1175/2009JAMC2227.1.

_ - M. B. Yeary, and G. Zhang, 2012: Efficient ways to learn weather radar polarimetry. IEEE Trans. Educ., 55, 58-68, https://doi.org/10.1109/TE.2011.2118211.

Carey, L. D., S. A. Rutledge, D. A. Ahijevych, and T. D. Keenan, 2000: Correcting propagation effects in C-band polarimetric radar observations of tropical convection using differential propagation phase. J. Appl. Meteor., 39, 1405-1433, https://doi. org/10.1175/1520-0450(2000)039<1405:CPEICB >2.0.CO;2.

Chen, H., V. Chandrasekar, and R. Bechini, 2017: An improved dual-polarization radar rainfall algorithm (DROPS2.0): Application in NASA IFloodS Field Campaign. J. Hydrometeor., 18, 917-937, https://doi.org/10.1175/JHM-D-16-0124.1.

Chen, X., K. Zhao, and M. Xue, 2014: Spatial and temporal characteristics of warm season convection over Pearl River Delta region, China, based on 3 years of operational radar data. J. Geophys. Res. Atmos., 119, 12 447-12 465, https://doi.org/ 10.1002/2014JD021965.

Cifelli, R., V. Chandrasekar, S. Lim, P. C. Kennedy, Y. Wang, and S. A. Rutledge, 2011: A new dual-polarization radar rainfall algorithm: Application in Colorado precipitation events. J. Atmos. Oceanic Technol., 28, 352-364, https://doi.org/10.1175/ 2010JTECHA1488.1.

Doviak, R. J., and D. S. Zrnić, 1993: Doppler Radar and Weather Observations. 2nd ed. Academic Press, 562 pp.

Giangrande, S. E., and A. V. Ryzhkov, 2005: Calibration of dualpolarization radar in the presence of partial beam blockage. J. Atmos. Oceanic Technol., 22, 1156-1166, https://doi.org/ 10.1175/JTECH1766.1.

- , and - 2008: Estimation of rainfall based on the results of polarimetric echo classification. J. Appl. Meteor. Climatol., 47, 2445-2462, https://doi.org/10.1175/2008JAMC1753.1.

- R. McGraw, and L. Lei, 2013: An application of linear programming to polarimetric radar differential phase processing. J. Atmos. Oceanic Technol., 30, 1716-1729, https://doi.org/ 10.1175/JTECH-D-12-00147.1.

Hogan, R. J., 2007: A variational scheme for retrieving rainfall rate and hail reflectivity fraction from polarization radar. J. Appl. Meteor. Climatol., 46, 1544-1564, https://doi.org/10.1175/ JAM2550.1.

Huang, H., G. Zhang, K. Zhao, and S. E. Giangrande, 2017: A hybrid method to estimate specific differential phase and rainfall with linear programming and physics constraints. IEEE Trans. Geosci. Remote Sens., 55, 96-111, https://doi.org/ 10.1109/TGRS.2016.2596295.

Hubbert, J., and V. N. Bringi, 1995: An iterative filtering technique for the analysis of copolar differential phase and dualfrequency radar measurements. J. Atmos. Oceanic Technol., 12, 643-648, https://doi.org/10.1175/1520-0426(1995)012<0643: AIFTFT $>2.0 . \mathrm{CO} ; 2$.

_ , V. Chandrasekar, V. N. Bringi, and P. Meischner, 1993: Processing and interpretation of coherent dual-polarized radar 
measurements. J. Atmos. Oceanic Technol., 10, 155-164, https://doi.org/10.1175/1520-0426(1993)010<0155:PAIOCD >2.0. $\mathrm{CO} ; 2$.

Kalnay, E., 2003: Atmospheric Modeling, Data Assimilation, and Predictability. Cambridge University Press, 368 pp.

Lee, G. W., 2006: Sources of errors in rainfall measurements by polarimetric radar: Variability of drop size distributions, observational noise, and variation of relationships between $\mathrm{R}$ and polarimetric parameters. J. Atmos. Oceanic Technol., 23, 1005-1028, https://doi.org/10.1175/JTECH1899.1.

Lee, J.-S., M. R. Grunes, and G. de Grandi, 1999: Polarimetric SAR speckle filtering and its implication for classification. IEEE Trans. Geosci. Remote Sens., 37, 2363-2373, https://doi.org/ 10.1109/36.789635.

Maesaka, T., K. Iwanami, and M. Maki, 2012: Non-negative $K_{\mathrm{DP}}$ estimation by monotone increasing $\Phi_{\mathrm{DP}}$ assumption below melting layer. Extended Abstracts, Seventh European Conf. on Radar in Meteorology and Hydrology (ERAD 2012), Toulouse, France, Météo-France, 26 QPE, http://www.meteo.fr/ cic/meetings/2012/ERAD/extended_abs/QPE_233_ext_abs.pdf.

Marshall, J. S., and W. M. K. Palmer, 1948: The distribution of raindrops with size. J. Meteor., 5, 165-166, https://doi.org/ 10.1175/1520-0469(1948)005<0165:TDORWS >2.0.CO;2.

Mishchenko, M. I., J. W. Hovenier, and L. D. Travis, 2000: Light Scattering by Nonspherical Particles. Academic Press, 690 pp.

Porcacchia, L., P. E. Kirstetter, J. J. Gourley, V. Maggioni, B. L. Cheong, and M. N. Anagnostou, 2017: Toward a polarimetric radar classification scheme for coalescencedominant precipitation: Application to complex terrain. J. Hydrometeor., 18, 3199-3215, https://doi.org/10.1175/ JHM-D-17-0016.1.

Rodgers, C. D., 2000: Inverse Methods for Atmospheric Sounding: Theory and Practice. Series on Atmospheric, Oceanic, and Planetary Physics, Vol. 2, World Scientific, 256 pp.

Ryzhkov, A. V., and D. Zrnić, 1996: Assessment of rainfall measurement that uses specific differential phase. J. Appl.
Meteor., 35, 2080-2090, https://doi.org/10.1175/1520-0450(1996) 035<2080:AORMTU>2.0.CO;2.

- S. E. Giangrande, and T. J. Schuur, 2005a: Rainfall estimation with a polarimetric prototype of WSR-88D. J. Appl. Meteor., 44, 502-515, https://doi.org/10.1175/JAM2213.1.

— T. J. Schuur, D. W. Burgess, P. L. Heinselman, S. E. Giangrande, and D. S. Zrnic, 2005b: The Joint Polarization Experiment: Polarimetric rainfall measurements and hydrometeor classification. Bull. Amer. Meteor. Soc., 86, 809-824, https://doi.org/ 10.1175/BAMS-86-6-809.

— M. Diederich, P. Zhang, and C. Simmer, 2014: Potential utilization of specific attenuation for rainfall estimation, mitigation of partial beam blockage, and radar networking. J. Atmos. Oceanic Technol., 31, 599-619, https://doi.org/ 10.1175/JTECH-D-13-00038.1.

Schönhuber, M., G. Lammer, and W. Randeu, 2007: One decade of imaging precipitation measurement by $2 \mathrm{D}$-videodistrometer. Adv. Geosci., 10, 85-90, https://doi.org/10.5194/ adgeo-10-85-2007.

Seliga, T. A., and V. N. Bringi, 1976: Potential use of radar differential reflectivity measurements at orthogonal polarizations for measuring precipitation. J. Appl. Meteor., 15, 69-76, https://doi.org/10.1175/1520-0450(1976)015<0069: PUORDR $>2.0 . C O ; 2$.

Steiner, M., R. A. Houze, and S. E. Yuter, 1995: Climatological characterization of three-dimensional storm structure from operational radar and rain gauge data. J. Appl. Meteor., 34, 1978-2007, https://doi.org/10.1175/1520-0450(1995)034<1978: CCOTDS $>2.0 . \mathrm{CO} ; 2$.

Sun, W., and Y.-X. Yuan, 2006: Optimization Theory and Methods: Nonlinear Programming. Springer Optimization and Its Applications, Vol. 1, Springer, $688 \mathrm{pp}$.

Wang, Y., and V. Chandrasekar, 2009: Algorithm for estimation of the specific differential phase. J. Atmos. Oceanic Technol., 26, 2565-2578, https://doi.org/10.1175/2009JTECHA1358.1.

Zhang, G., 2016: Weather Radar Polarimetry. CRC Press, 322 pp. 